\title{
On complete monotonicity for several classes of functions related to ratios of gamma functions
}

\author{
Feng $\mathrm{Qi}^{1,2,3^{*}}$ (D) and Ravi P. Agarwal ${ }^{4,5}$
}

\section{"Correspondence:}

qifeng618@hotmail.com

${ }^{1}$ Institute of Mathematics, Henan

Polytechnic University, Jiaozuo,

China

${ }^{2}$ College of Mathematics, Inner

Mongolia University for

Nationalities, Tongliao, China

Full list of author information is

available at the end of the article

\begin{abstract}
Let $\Gamma(x)$ denote the classical Euler gamma function. The logarithmic derivative $\psi(x)=[\ln \Gamma(x)]^{\prime}=\frac{\Gamma^{\prime}(x)}{\Gamma(x)}, \psi^{\prime}(x)$, and $\psi^{\prime \prime}(x)$ are, respectively, called the digamma, trigamma, and tetragamma functions. In the paper, the authors survey some results related to the function $\left[\psi^{\prime}(x)\right]^{2}+\psi^{\prime \prime}(x)$, its $q$-analogs, its variants, its divided difference forms, several ratios of gamma functions, and so on. These results include the origins, positivity, inequalities, generalizations, completely monotonic degrees, (logarithmically) complete monotonicity, necessary and sufficient conditions, equivalences to inequalities for sums, applications, and the like. Finally, the authors list several remarks and pose several open problems.
\end{abstract}

MSC: Primary 33B15; secondary 26A48; 26A51; 26D10; 26D15; 33D05; 44A10

Keywords: Digamma function; Trigamma function; Tetragamma function; Polygamma function; Ratio of gamma functions; $q$-analog; Complete monotonicity; Completely monotonic degree; Logarithmically completely monotonic function; Divided difference; Necessary and sufficient condition; Inequality; Generalization; Open problem; Application

\section{Preliminaries}

In order to proceed fluently and smoothly, we prepare some definitions, concepts, and notation in this section.

\subsection{Logarithmically completely monotonic functions}

Recall from [55, Chapter XIII], [122, Chap. 1], and [127, Chapter IV] that an infinitely differentiable function $f(x)$ is said to be completely monotonic on an interval $I \subseteq \mathbb{R}$ if $0 \leq(-1)^{n-1} f^{(n-1)}(x)<\infty$ for $x \in I$ and $n \in \mathbb{N}$. A characterization of completely monotonic functions is given by the Bernstein-Widder theorem [127, p. 160, Theorem 12a], which states that a function $f(x)$ on $[0, \infty)$ is completely monotonic if and only if there exists a bounded and non-decreasing function $\alpha(t)$ such that the integral

$$
f(x)=\int_{0}^{\infty} e^{-x t} \mathrm{~d} \alpha(t)
$$

(c) The Author(s) 2019. This article is distributed under the terms of the Creative Commons Attribution 4.0 International License (http://creativecommons.org/licenses/by/4.0/), which permits unrestricted use, distribution, and reproduction in any medium, provided you give appropriate credit to the original author(s) and the source, provide a link to the Creative Commons license, and indicate if changes were made. 
converges for $x \in[0, \infty)$. Equivalently speaking, a function $f(x)$ is completely monotonic on $[0, \infty)$ if and only if it is a Laplace transform of a bounded and non-decreasing measure $\alpha(t)$. This is one of many reasons why mathematicians have been studying the class of completely monotonic functions for so many years.

Recall from [122, Chap. 2] that if $f:(0, \infty) \rightarrow[0, \infty)$ can be written as

$$
f(x)=\frac{a}{x}+b+\int_{0}^{\infty} \frac{1}{s+x} \mathrm{~d} \mu(s), \quad a, b \geq 0
$$

where $\mu$ is a measure on $(0, \infty)$ such that $\int_{0}^{\infty} \frac{1}{1+s} \mathrm{~d} \mu(s)<\infty$, then we call $f$ a Stieltjes transform.

Recall from $[11,85,98]$ that a positive and infinitely differentiable function $f(x)$ is said to be logarithmically completely monotonic on $I \subseteq \mathbb{R}$ if its $\operatorname{logarithm} \ln f(x)$ satisfies $0 \leq$ $(-1)^{k}[\ln f(x)]^{(k)}<\infty$ for $x \in I$ and $k \in \mathbb{N}$.

Definition 1.2 in [36] states that a function $f: I \rightarrow[0, \infty)$ is called a Bernstein function on $I$ if $f^{\prime}(t)$ is completely monotonic on $I$. This class of functions can be characterized by [122, Theorem 3.2] which states that $f:(0, \infty) \rightarrow[0, \infty)$ is a Bernstein function if and only if it admits the representation

$$
f(x)=\alpha+\beta x+\int_{0}^{\infty}\left(1-e^{-x t}\right) \mathrm{d} \mu(t)
$$

where $\alpha, \beta \geq 0$ and $\mu$ is a positive measure satisfying $\int_{0}^{\infty} \min \{1, t\} \mathrm{d} \mu(t)<\infty$.

Among the above classes of functions, there exist the following relations:

1. the set of all logarithmically completely monotonic functions on $I$ is a strict subset of all completely monotonic functions on $I$; see [85, Theorem 1$]$;

2. the set of all Stieltjes transforms is a strict subset of all logarithmically completely monotonic functions on $(0, \infty)$; see [15, Theorem 1.2];

3. the reciprocal of a Bernstein function is logarithmically completely monotonic; see [21, pp. 161-162, Theorem 3] and [122, p. 64, Proposition 5.25].

For more information on these relations, please refer to $[15,27,85,98,100,101,108,122]$ and the closely related references therein. This is one of many reasons why mathematicians have been studying the class of logarithmically completely monotonic functions for such a long time.

Let $f(x)$ be completely monotonic on $(0, \infty)$ and $f(\infty)=\lim _{x \rightarrow \infty} f(x)$. Recall from [32, $36,47-51,75,94,97,102,103,116,119,120]$ that, if for some $r \in \mathbb{R}$ the function $x^{r}[f(x)-f(\infty)]$ is completely monotonic on $(0, \infty)$, but for any positive number $\varepsilon>0$ the function $x^{r+\varepsilon}[f(x)-f(\infty)]$ is not, then the number $r$ is called the completely monotonic degree of $f(x)$ with respect to $x \in(0, \infty)$; if $x^{r}[f(x)-f(\infty)]$ is completely monotonic on $(0, \infty)$ for all $r \in \mathbb{R}$, then the completely monotonic degree of $f(x)$ with respect to $x \in(0, \infty)$ is said to be $\infty$. For convenience, the notation $\operatorname{deg}_{\mathrm{cm}}^{x}[f(x)]$ was designed in [32] to denote the completely monotonic degree $r$ of $f(x)$ with respect to $x \in(0, \infty)$. This notion can help to measure completely monotonic functions more accurately and precisely. Theorem 1.1 in [49], Theorem 1.3 in [50], and Proposition 1.2 in [102] can be modified as that $\operatorname{deg}_{\mathrm{cm}}^{x}[f(x)]=\alpha>0$ if and only if

$$
f(x)-f(\infty)=\int_{0}^{\infty} e^{-x t} I_{\alpha}(m)(t) \mathrm{d} t
$$


where

$$
I_{\alpha}(m)(t)=\frac{1}{\Gamma(\alpha)} \int_{0}^{t}(t-s)^{\alpha-1} \mathrm{~d} m(s)
$$

is called the Riemann-Liouville fractional integral of order $\alpha$ for a bounded and nondecreasing Borel measure $m$ on $[0, \infty)$. For information on the Riemann-Liouville fractional integrals, please refer to $[114,126]$ and the closely related references therein.

\subsection{The gamma and polygamma functions and their $q$-analogs}

The classical Euler gamma function $\Gamma(z)$ can be defined by an improper integral

$$
\Gamma(z)=\int_{0}^{\infty} t^{z-1} e^{-t} \mathrm{~d} t, \quad \Re(z)>0,
$$

or by the limit

$$
\Gamma(z)=\lim _{n \rightarrow \infty} \frac{n ! n^{z}}{\prod_{k=0}^{n}(z+k)}, \quad z \neq 0,-1,-2, \ldots
$$

For more information, please refer to [10, Chap. 1] and [66, 71, 90, 92]. The logarithmic derivative of $\Gamma(z)$, denoted by $\psi(z)=\frac{\Gamma^{\prime}(z)}{\Gamma(z)}$, is called the digamma function. The derivatives $\psi^{\prime}(z)$ and $\psi^{\prime \prime}(z)$ are called the trigamma and tetragamma functions, respectively. As a whole, the functions $\psi^{(i)}(z)$ for $i \geq 0$ are called polygamma functions.

The $q$-analog $\Gamma_{q}(z)$ of the gamma function $\Gamma(z)$ can be defined for $\Re(z)>0$ by

$$
\Gamma_{q}(z)= \begin{cases}(1-q)^{1-z} \prod_{i=0}^{\infty} \frac{1-q^{i+1}}{1-q^{i+z}}, & 0<q<1 \\ (q-1)^{1-z} q^{\left(\frac{z}{2}\right)} \prod_{i=0}^{\infty} \frac{1-q^{-(i+1)}}{1-q^{-(i+z)}}, & q>1 .\end{cases}
$$

The $q$-digamma function $\psi_{q}(z)$, the $q$-analog of the digamma function $\psi(z)$, can be defined by

$$
\psi_{q}(z)=\frac{\Gamma_{q}^{\prime}(z)}{\Gamma_{q}(z)}= \begin{cases}-\ln (1-q)+\ln q \sum_{k=0}^{\infty} \frac{q^{k+z}}{1-q^{k+z}}, & 0<q<1 ; \\ -\ln (q-1)+\ln q\left(z-\frac{1}{2}-\sum_{k=0}^{\infty} \frac{1}{q^{k+z}-1}\right), & q>1 .\end{cases}
$$

These functions satisfy the relations

$$
\Gamma_{q}(z)=q^{\left(\begin{array}{c}
z-1 \\
2
\end{array}\right)} \Gamma_{1 / q}(z), \quad \lim _{q \rightarrow 1^{ \pm}} \Gamma_{q}(z)=\Gamma(z), \quad \lim _{q \rightarrow 1^{ \pm}} \psi_{q}(z)=\psi(z) .
$$

The proofs of the above two limits can be found in [9, Appendix A], [10, pp. 493-496], and [46, Appendix B]. The functions $\psi_{q}^{(k)}(z)$, the $q$-analogs of the polygamma functions $\psi^{(k)}(z)$, for $k \geq 0$, are called the $q$-polygamma functions.

Equation (1.11) in [42] and its corrected version [43] read

$$
\psi_{q}(x)=-\ln (1-q)-\int_{0}^{\infty} \frac{e^{-x t}}{1-e^{-t}} \mathrm{~d} \gamma_{q}(t), \quad 0<q<1, x>0,
$$


where

$$
\gamma_{q}(t)= \begin{cases}-\ln q \sum_{k=1}^{\infty} \delta(t+k \ln q), & 0<q<1 \\ t, & q=1\end{cases}
$$

and $\delta(t)$ represents the Dirac delta function, that is, $\mathrm{d} \gamma_{q}(t)$ is a discrete measure with positive masses $|\ln q|$ at the positive points $k|\ln q|$ for $k \in \mathbb{N}$. Accordingly, we obtain

$$
\int_{0}^{\infty} e^{-x t} \mathrm{~d} \gamma_{q}(t)=-\frac{q^{x} \ln q}{1-q^{x}} \quad \text { and } \quad \int_{0}^{\infty} t e^{-x t} \mathrm{~d} \gamma_{q}(t)=\frac{q^{x}(\ln q)^{2}}{\left(1-q^{x}\right)^{2}}
$$

for $0<q<1$ and $x>0$. Differentiating (1.4) with respect to $x$ yields

$$
\psi_{q}^{(k)}(x)=(-1)^{k+1} \int_{0}^{\infty} \frac{t^{k} e^{-x t}}{1-e^{-t}} \mathrm{~d} \gamma_{q}(t), \quad 0<q<1, k \in \mathbb{N} .
$$

In [26, p. 1245, Theorem 4.4, (4.15)], [67, Lemma 2.3 and Remark 2.1], [64, Theorem 7.2, (7.5)], and [106, p. 152, Theorem 4.22, (4.20)], the identity

$$
\psi_{q}^{(k-1)}(x)-\psi_{q}^{(k-1)}(x+1)=(\ln q) \frac{\mathrm{d}^{k-1}}{\mathrm{~d} x^{k-1}}\left(\frac{q^{x}}{1-q^{x}}\right), \quad 0<q<1
$$

was presented for $x \in(0, \infty)$ and $k \in \mathbb{N}$. One can also find this knowledge in [25, 41, 42, $56,64,67,73,105]$ and the closely related references therein.

\subsection{Divided differences}

The divided difference $f\left[x_{1}, x_{2}, \ldots, x_{n}\right]$ of a function $f(x)$ on $n$ points $x_{1}, x_{2}, \ldots, x_{n}$ is defined by $f\left[x_{1}\right] \equiv f\left(x_{1}\right)$ and

$$
f\left[x_{1}, x_{2}, \ldots, x_{n}\right]=\frac{f\left[x_{1}, \ldots, x_{n-1}\right]-f\left[x_{2}, \ldots, x_{n}\right]}{x_{1}-x_{n}}
$$

for $n \geq 2$. In particular, for $n=2$,

$$
f\left[x_{1}, x_{2}\right]=\frac{f\left(x_{1}\right)-f\left(x_{2}\right)}{x_{1}-x_{2}}=\frac{1}{x_{1}-x_{2}} \int_{x_{1}}^{x_{2}} f^{\prime}(t) \mathrm{d} t,
$$

if $f$ is differentiable. See [1, p. 877, 2.5.14] and [59, p. 23]. On taking $f(x)=\psi^{(i)}(x)$ in (1.5), the divided differences of the polygamma functions are

$$
\psi^{(i)}\left[x_{1}, x_{2}\right]=\frac{\psi^{(i)}\left(x_{1}\right)-\psi^{(i)}\left(x_{2}\right)}{x_{1}-x_{2}}=\frac{1}{x_{1}-x_{2}} \int_{x_{1}}^{x_{2}} \psi^{(i+1)}(u) \mathrm{d} u
$$

for $x_{1}, x_{2} \in(0, \infty)$. By the way, we note that

$$
A(a, b ; f)=\frac{1}{b-a} \int_{a}^{b} f(t) \mathrm{d} t
$$

is called the arithmetic mean of the function $f$ on the interval $[a, b]$; see $[18, \mathrm{p} .368]$ and [117]. 


\section{Three origins}

The topic we are about to discuss in this paper have three main origins, as discussed now.

\subsection{The first origin}

Let $s, t \in \mathbb{R}$ and define

$$
z_{s, t}(x)=W_{s, t}(x)-x= \begin{cases}{\left[\frac{\Gamma(x+s)}{\Gamma(x+t)}\right]^{1 /(s-t)}-x,} & s \neq t, \\ e^{\psi(x+s)}-x, & s=t,\end{cases}
$$

for $x \in(-\min \{s, t\}, \infty)$. The monotonicity and convexity of $z_{s, t}(x)$ have been studied for a long time. An easy computation yields

$$
\frac{z_{s, t}^{\prime \prime}(x)}{z_{s, t}(x)+x}=\left[\frac{\psi(x+t)-\psi(x+s)}{t-s}\right]^{2}+\frac{\psi^{\prime}(x+t)-\psi^{\prime}(x+s)}{t-s} \triangleq \Delta_{s, t}(x)
$$

for $s \neq t$ and $t-s \neq \pm 1$. In [23, pp. 243-245], by using the representation

$$
\psi(x+1)=-\gamma+\sum_{k=1}^{\infty}\left(\frac{1}{k}-\frac{1}{x+k}\right)
$$

and the elementary algebraic inequality

$$
\frac{1}{a b}+\frac{1}{c d}>\frac{1}{a c}+\frac{1}{b d}
$$

for $a \leq b<c \leq d$, the positivity for $0<|t-s|<1$ and negativeness for $|t-s|>1$ of the function $\Delta_{s, t}(x)$ were proved. Consequently, it was proved in [23, Theorem 1] that the function $z_{s, t}(x)$ is

1. convex and decreasing for $|t-s|<1$, or

2. concave and increasing for $|t-s|>1$.

Hereafter, several alternative proofs for [23, Theorem 1] were supplied in $[19,30,31,88$, 99]. The differences among $[19,23,30,31,88,99]$ are the manners of and the approaches to coping with the positivity or negativity of the function $\Delta_{s, t}(x)$. For more information, please refer to the expository articles $[64,69,106,107]$ and the references therein.

We note that, since

$$
\ln W_{s, t}(x)=\frac{\ln \Gamma(x+s)-\ln \Gamma(x+t)}{s-t}=\frac{1}{s-t} \int_{t}^{s} \psi(x+u) \mathrm{d} u,
$$

the function $\frac{1}{W_{s, t}(x)}$ is logarithmically completely monotonic on $(-\min \{s, t\}, \infty)$.

We guess that the function $W_{s, t}(x)$ is a Bernstein function on $(-\min \{s, t\}, \infty)$.

\subsection{The second origin}

In [3, p. 208, (4.39)], to show that the double inequality

$$
(n-1) ! \exp \left[\frac{\alpha}{x}-n \psi(x)\right]<\left|\psi^{(n)}(x)\right|<(n-1) ! \exp \left[\frac{\beta}{x}-n \psi(x)\right]
$$


for $x>0$ and $n \in \mathbb{N}$ is valid if and only if $\alpha \leq-n$ and $\beta \geq 0$, a single-sided inequality,

$$
\begin{aligned}
\Delta(x) \triangleq & {\left[\psi^{\prime}(x)\right]^{2}+\psi^{\prime \prime}(x) } \\
>> & \frac{p(x)}{900 x^{4}(x+1)^{10}}=\frac{1}{2 x^{4}}-\frac{1}{x^{3}}+\frac{34}{15 x^{2}}-\frac{14}{3 x}+\frac{14}{3(x+1)} \\
& +\frac{12}{5(x+1)^{2}}+\frac{17}{15(x+1)^{3}}+\frac{9}{20(x+1)^{4}}+\frac{1}{10(x+1)^{5}} \\
& -\frac{7}{180(x+1)^{6}}-\frac{1}{30(x+1)^{7}}-\frac{1}{90(x+1)^{8}}+\frac{1}{900(x+1)^{10}}, \quad x>0,
\end{aligned}
$$

was established, where

$$
\begin{aligned}
p(x)= & 75 x^{10}+900 x^{9}+4840 x^{8}+15,370 x^{7}+31,865 x^{6}+45,050 x^{5} \\
& +44,101 x^{4}+29,700 x^{3}+13,290 x^{2}+3600 x+450 .
\end{aligned}
$$

By the way, by the same technique as in [3, p. 208, (4.39)], an upper bound

$$
\begin{aligned}
\Delta(x)< & \frac{36+180 x+408 x^{2}+504 x^{3}+352 x^{4}+132 x^{5}+21 x^{6}}{36 x^{4}(1+x)^{6}} \\
= & \frac{1}{x^{4}}-\frac{1}{x^{3}}+\frac{7}{3 x^{2}}-\frac{5}{x}+\frac{5}{x+1}+\frac{8}{3(x+1)^{2}} \\
& +\frac{4}{3(x+1)^{3}}+\frac{7}{12(x+1)^{4}}+\frac{1}{6(x+1)^{5}}+\frac{1}{36(x+1)^{6}}, \quad x>0,
\end{aligned}
$$

was claimed in [72].

The double inequality (2.3) can be restated as follows: the double inequality

$$
\exp \left[\frac{\alpha}{x}-\psi(x)\right]<\sqrt[n]{\frac{\left|\psi^{(n)}(x)\right|}{(n-1) !}}<\exp \left[\frac{\beta}{x}-\psi(x)\right]
$$

for $x>0$ and $n \in \mathbb{N}$ is valid if and only if $\alpha \leq-1$ and $\beta \geq 0$.

\subsection{The third origin}

In [13, Lemma 1.1], a weaker inequality,

$$
\Delta(x)>0, \quad x>0,
$$

than (2.4) was recovered. The sketch of the proof for (2.6) is as follows. By virtue of (3.2), one can find

$$
\Delta(x)-\Delta(x+1)=\frac{2}{x^{2}}\left[\psi^{\prime}(x)-\frac{1}{x}-\frac{1}{2 x^{2}}\right]>0, \quad x>0 .
$$

This implies the inequality (2.6). In fact, since (5.1), this also implies complete monotonicity of $\Delta(x)$ on $(0, \infty)$.

\subsection{A connection between three origins}

It is easy to observe that $\lim _{s, t \rightarrow 0} \Delta_{s, t}(x)=\Delta(x)$. Conversely, it is also obvious that the function $\Delta_{s, t}(x)$ is a divided difference form of the function $\Delta(x)$. 


\section{Generalizations of the second and third origins}

We now begin to collect some generalizations related to the second and third origins.

\subsection{Some basic tools}

One of the difficulties to generalize the above results is how to meaningfully compute any high order derivatives of the term $\left[\psi^{\prime}(x)\right]^{2}$. If differentiating this term directly and consecutively, the derivatives would become more and more complicated and one cannot read a very useful message from these derivatives.

To overcome the above-mentioned difficulty and to generalize the above three original results, we will employ mathematical induction and the following basic, well-known, and very effectual tools.

1. Let $f(x)$ be defined on an infinite interval $I$ whose right endpoint is $\infty$. If

$$
\lim _{x \rightarrow \infty} f(x)=\epsilon \text { and } f(x)-f(x+\varepsilon)>0
$$

for some given $\varepsilon>0$, then $f(x)>\epsilon$ on $I$. See [13, Lemma 1.1], [30, Lemma 1], [28, p. 223, Lemma 2.1], [29, p. 107, Lemma 4], [62, p. 526, Lemma 2.1], [88, p. 1981, Lemma 2.5], [100, p. 2155, Lemma 1], [108, Lemma 2.1], [38, 89, 107] and closely related references therein. This has been summarized in [76, Remark 1, Theorem 3] which states that a function $f(x)$ on an infinite interval $I$ whose right endpoint is $\infty$ is completely monotonic on $I$ if and only if

(a) there exist positive numbers $\varepsilon$ such that the differences

$$
(-1)^{i}[f(x)-f(x+\varepsilon)]^{(i)}=(-1)^{i}\left[f^{(i)}(x)-f^{(i)}(x+\varepsilon)\right]
$$

are nonnegative for all integers $i \geq 0$ on $I$;

(b) the limits

$$
\lim _{x \rightarrow \infty}\left[(-1)^{i} f^{(i)}(x)\right]=(-1)^{i} \lim _{x \rightarrow \infty} f^{(i)}(x)=\epsilon_{i} \geq 0
$$

exist for all integers $i \geq 0$.

Since the gamma function $\Gamma(x)$ and polygamma functions $\psi^{(i)}(x)$ for $i \geq 0$ are of the recurrent period 1, that is, the recurrence relations $\Gamma(x+1)=x \Gamma(x)$ and (3.2) hold, when dealing with some problems related to the gamma and polygamma functions $\Gamma(x)$ and $\psi^{(i)}(x)$, one can select $\varepsilon=1$ for possibly simplifying computation.

2. For $x>0, n \in \mathbb{N}$, and $r>0$, we have

$$
\begin{aligned}
& \psi^{(n-1)}(x+1)=\psi^{(n-1)}(x)+\frac{(-1)^{n-1}(n-1) !}{x^{n}}, \\
& \psi^{(n)}(x)=(-1)^{n+1} \int_{0}^{\infty} \frac{t^{n}}{1-e^{-t}} e^{-x t} \mathrm{~d} t
\end{aligned}
$$

and

$$
\frac{1}{x^{r}}=\frac{1}{\Gamma(r)} \int_{0}^{\infty} t^{r-1} e^{-x t} \mathrm{~d} t
$$

See [1, pp. 255-260] or other monographs and handbooks for special functions. 
3. A product of finitely many completely monotonic functions on an interval $I$ is also completely monotonic on $I$. See [122, Corollary 1.6].

\subsection{Complete monotonicity}

In [40, Theorem 1], the function

$$
f_{1}(x)=\Delta(x)-\frac{p(x)}{900 x^{4}(x+1)^{10}}
$$

which was constructed from the inequality (2.4), was proved to be completely monotonic on $(0, \infty)$.

Sketch of the proof Making use of (3.2) yields

$$
f_{1}(x)-f_{1}(x+1)=\frac{2}{x^{2}}\left[\psi^{\prime}(x)-\frac{Q_{21}(x)}{1800 x^{2}(1+x)^{10}(2+x)^{10}}\right] \triangleq \frac{2}{x^{2}} H_{1}(x),
$$

where $Q_{21}(x)$ is a polynomial of degree 21 with positive coefficients. Employing (3.3) and (3.4), one can prove that $H_{1}(x)$ is a completely monotonic function on $(0, \infty)$. Hence, the function $f_{1}(x)-f_{1}(x+1)$ is completely monotonic on $(0, \infty)$. As a result, it follows that

$$
(-1)^{k}\left[f_{1}(x)-f_{1}(x+1)\right]^{(k)}=(-1)^{k} f_{1}^{(k)}(x)-(-1)^{k} f_{1}^{(k)}(x+1) \geq 0
$$

and, by (3.1), that

$$
\begin{aligned}
(-1)^{k} f_{1}^{(k)}(x) & \geq(-1)^{k} f_{1}^{(k)}(x+1) \geq(-1)^{k} f_{1}^{(k)}(x+2) \geq \cdots \\
& \geq(-1)^{k} f_{1}^{(k)}(x+m) \geq \cdots \geq \lim _{m \rightarrow \infty}\left[(-1)^{k} f_{1}^{(k)}(x+m)\right]=0
\end{aligned}
$$

for $k \geq 0$ on $(0, \infty)$. The required result is thus proved.

\subsection{A double inequality and complete monotonicity}

The double inequality constituted by (2.4) and (2.5) was simplified in [136, Theorem 1] as

$$
\begin{aligned}
\frac{1}{x^{4}} & -\frac{2}{x^{3}}+\frac{37}{12 x^{2}}-\frac{25}{6 x}+\frac{25}{6(x+1)}+\frac{13}{12(x+1)^{2}} \\
& =\frac{x^{2}+12}{12 x^{4}(x+1)^{2}}<\Delta(x) \\
& <\frac{x+12}{12 x^{4}(x+1)}=\frac{1}{x^{4}}-\frac{23}{12 x^{3}}+\frac{17}{6 x^{2}}-\frac{15}{4 x}+\frac{15}{4(x+1)}+\frac{11}{12(x+1)^{2}}
\end{aligned}
$$

on $(0, \infty)$. More strongly, the functions

$$
f_{2}(x)=\Delta(x)-\frac{x^{2}+12}{12 x^{4}(x+1)^{2}} \quad \text { and } \quad f_{3}(x)=\frac{x+12}{12 x^{4}(x+1)}-\Delta(x)
$$

were proved in [136, Theorem 2] to be completely monotonic on $(0, \infty)$. 
Sketch of the proof By (3.2), we can obtain

$$
f_{2}(x)-f_{2}(x+1)=\frac{2}{x^{2}}\left[\psi^{\prime}(x)-\frac{Q_{7}(x)}{6 x^{2}(1+x)^{4}(2+x)^{2}}\right] \triangleq \frac{2}{x^{2}} H_{2}(x)
$$

and

$$
f_{3}(x)-f_{3}(x+1)=\frac{2}{x^{2}}\left[\frac{Q_{6}(x)}{24 x^{2}(x+1)^{4}(x+2)}-\psi^{\prime}(x)\right] \triangleq \frac{2}{x^{2}} H_{3}(x),
$$

where $Q_{6}(x)$ and $Q_{7}(x)$ are, respectively, polynomials of degree 6 and 7 with positive coefficients. Utilizing (3.3) and (3.4), one can prove that the functions $H_{2}(x)$ and $H_{3}(x)$ are completely monotonic on $(0, \infty)$. Finally, by $(3.1)$, one can conclude that the functions $f_{2}(x)$ and $f_{3}(x)$ are completely monotonic on $(0, \infty)$.

\subsection{Necessary and sufficient conditions}

For $\lambda \in \mathbb{R}$, let

$$
h_{\lambda}(x)=\Delta(x)-\frac{x^{2}+\lambda x+12}{12 x^{4}(x+1)^{2}}
$$

on $(0, \infty)$. This is a generalization of the function $f_{2}(x)$ in $(3.5)$. In [72, Theorem 1$]$, it was established that

1. the function $h_{\lambda}(x)$ is completely monotonic on $(0, \infty)$ if and only if $\lambda \leq 0$;

2. the function $-h_{\lambda}(x)$ is completely monotonic on $(0, \infty)$ if and only if $\lambda \geq 4$;

3. consequently, the double inequality

$$
\frac{x^{2}+\mu x+12}{12 x^{4}(x+1)^{2}}<\Delta(x)<\frac{x^{2}+v x+12}{12 x^{4}(x+1)^{2}}
$$

holds on $(0, \infty)$ if and only if $\mu \leq 0$ and $v \geq 4$.

Sketch of the proofs It is easy to see that $h_{\lambda}(x)=f_{2}(x)-\lambda g(x)$ and $g(x)=\frac{1}{12 x^{3}(x+1)^{2}}$. Since $f_{2}(x)$ and $g(x)$ are completely monotonic functions on $(0, \infty)$, when $\lambda \leq 0$, the function $h_{\lambda}(x)$ is also completely monotonic on $(0, \infty)$. Utilizing (3.2) for $n=1$ and $n=2$ yields

$$
\begin{aligned}
h_{\lambda}(x)= & -\frac{\lambda}{12}\left[\frac{1}{x^{3}}-\frac{2}{x^{2}}+\frac{3}{x}-\frac{4+3 x}{(1+x)^{2}}\right]-\frac{37}{12 x^{2}}+\frac{25}{6 x}-\frac{63+50 x}{12(1+x)^{2}} \\
& +\frac{2}{x^{2}} \psi^{\prime}(x+1)+\left[\psi^{\prime}(x+1)\right]^{2}+\psi^{\prime \prime}(x+1) .
\end{aligned}
$$

Therefore, if $\lambda>0$ then $\lim _{x \rightarrow 0^{+}} h_{\lambda}(x)=-\infty$. This implies that the function $h_{\lambda}(x)$ is completely monotonic on $(0, \infty)$ if and only if $\lambda \leq 0$.

From (3.2), (3.3), and (3.4), we can arrive at

$$
\begin{aligned}
h_{\lambda}(x)-h_{\lambda}(x+1) & =\frac{2}{x^{2}}\left[\psi^{\prime}(x)-\frac{\lambda\left(4+8 x+5 x^{2}\right)}{24 x(1+x)^{3}(2+x)^{2}}-\frac{Q_{7}(x)}{6 x^{2}(1+x)^{4}(2+x)^{2}}\right] \\
& =\frac{1}{24 x^{2}} \int_{0}^{\infty}\left[\frac{4 P(t)}{Q(t)}-\lambda\right] Q(t) e^{-(x+2) t} \mathrm{~d} t,
\end{aligned}
$$


where

$$
\begin{aligned}
& P(t)=\frac{e^{2 t}\left(t^{3}-12 t^{2}+54 t-86\right)-e^{t}\left(t^{3}-12 t^{2}+16 t-160\right)-26 t-74}{e^{t}-1}, \\
& Q(t)=2 e^{2 t}-e^{t}\left(t^{2}-6 t+18\right)+8(t+2)>0, \\
& \lim _{t \rightarrow 0} \frac{P(t)}{Q(t)}=1, \quad \lim _{t \rightarrow \infty} \frac{P(t)}{Q(t)}=0,
\end{aligned}
$$

and $\frac{P(x)}{Q(x)}$ is strictly decreasing on $(0, \infty)$. By the Bernstein-Widder theorem stated in (1.1), it follows that

1. the difference $h_{\lambda}(x)-h_{\lambda}(x+1)$ is completely monotonic on $(0, \infty)$ if and only if $\lambda \leq 0$;

2. the difference $-\left[h_{\lambda}(x)-h_{\lambda}(x+1)\right]$ is completely monotonic on $(0, \infty)$ if and only if $\lambda \geq 4$.

Finally, by (3.1), one can conclude necessary and sufficient conditions for the function $h_{\lambda}(x)$ to be completely monotonic on $(0, \infty)$.

How much are completely monotonic degrees of the function $h_{\lambda}(x)$ for $\lambda \leq 0$ and its negative $-h_{\lambda}(x)$ for $\lambda \geq 4$ with respect to $x \in(0, \infty)$ ?

\subsection{A generalization of the third origin}

Lemma 1.2 in [14] shows that the inequality

$$
(-1)^{n} \psi^{(n+1)}(x)<\frac{n}{\sqrt[n]{(n-1) !}}\left[(-1)^{n-1} \psi^{(n)}(x)\right]^{1+1 / n}
$$

holds for $x>0$ and $n \in \mathbb{N}$. Remark 1.3 in [14] points out that the inequality (2.6) is the special case $n=1$ of the inequality (3.6). The inequality (3.6) was reformulated in [39, p. 108] as the form

$$
\sqrt[n+1]{\frac{\left|\psi^{(n+1)}(x)\right|}{n !}}<\sqrt[n]{\frac{\left|\psi^{(n)}(x)\right|}{(n-1) !}}
$$

for $n \in \mathbb{N}$ on $(0, \infty)$.

Sketch of the proof Theorem 2.1 in [8] states that the function

$$
F_{n, \alpha}(x)=\left[\psi^{(n)}(x)\right]^{2}-\alpha \psi^{(n-1)}(x) \psi^{(n+1)}(x), \quad n \geq 2
$$

is completely monotonic on $(0, \infty)$ if and only if $\alpha \leq \frac{n-1}{n}$, while its negative $-F_{n, \beta}(x)$ is completely monotonic on $(0, \infty)$ if and only if $\beta \geq \frac{n}{n+1}$. Consequently, a sharp double inequality

$$
\frac{n-1}{n}<\frac{\left[\psi^{(n)}(x)\right]^{2}}{\psi^{(n-1)}(x) \psi^{(n+1)}(x)}<\frac{n}{n+1}, \quad n \geq 2
$$

was derived in [8, Corollary 2.3]. In [14, Lemma 1.2], by virtue of the left inequality in (3.8), the function $\frac{\left[\psi^{(n)}(x)\right]^{n+1}}{\left[\psi^{(n+1)}(x)\right]^{n}}$ for $n \in \mathbb{N}$ was proved to be increasing on $(0, \infty)$. As a result, the inequality (3.6) was deduced straightforwardly. 


\subsection{Necessary and sufficient conditions for divided differences}

For $n \in \mathbb{N}$ and $p, q \in \mathbb{R}$, let

$$
\begin{aligned}
\phi_{n}(x) & = \begin{cases}-\frac{\left|\psi^{(n-1)}(x+p)\right|-\left|\psi^{(n-1)}(x+q)\right|}{p-q}, & p \neq q, \\
\left|\psi^{(n)}(x+q)\right|, & p=q,\end{cases} \\
& = \begin{cases}\frac{1}{p-q} \int_{q}^{p}\left|\psi^{(n)}(x+t)\right| \mathrm{d} t, & p \neq q, \\
\left|\psi^{(n)}(x+q)\right|, & p=q,\end{cases}
\end{aligned}
$$

and

$$
\Phi_{n, \lambda}(x)=\left[\phi_{n+1}(x)\right]^{2}-\lambda \phi_{n}(x) \phi_{n+2}(x) .
$$

In [128], Theorem 1 states that

1. when $p=q$,

(a) the function $\Phi_{n, \lambda}(x)$ is completely monotonic on $(-\min \{p, q\}, \infty)$ if and only if $\lambda \leq \frac{n}{n+1}$

(b) the function $-\Phi_{n, \lambda}(x)$ is completely monotonic on $(-\min \{p, q\}, \infty)$ if and only if $\lambda \geq \frac{n+1}{n+2} ;$

2. when $0<|p-q|<1$,

(a) the function $\Phi_{n, \lambda}(x)$ is completely monotonic on $(-\min \{p, q\}, \infty)$ if and only if $\lambda \leq \frac{n}{n+1}$

(b) the function $-\Phi_{n, \lambda}(x)$ is completely monotonic on $(-\min \{p, q\}, \infty)$ if and only if $\lambda \geq \sup _{t \in(0, \infty)} Q_{|p-q|}(t)$, where

$$
\begin{aligned}
& Q_{\alpha}(t)=\frac{\int_{0}^{1}\left(1-v^{2}\right)^{n} P_{\alpha}(t, v) \mathrm{d} v}{\int_{0}^{1}\left(1+v^{2}\right)\left(1-v^{2}\right)^{n-1} P_{\alpha}(t, v) \mathrm{d} v}, \\
& P_{\alpha}(t, v)=h_{\alpha}\left(\frac{1+v}{2} t\right) h_{\alpha}\left(\frac{1-v}{2} t\right),
\end{aligned}
$$

and

$$
h_{\alpha}(t)= \begin{cases}\frac{1}{\alpha} \frac{1-e^{-\alpha t}}{1-e^{-t}}, & \alpha \neq 0 \\ \frac{t}{1-e^{-t}}, & \alpha=0\end{cases}
$$

3. when $|p-q|>1$,

(a) the function $\Phi_{n, \lambda}(x)$ is completely monotonic on $(-\min \{p, q\}, \infty)$ if and only if $\lambda \leq \inf _{t \in(0, \infty)} Q_{|p-q|}(t)$;

(b) the function $-\Phi_{n, \lambda}(x)$ is completely monotonic on $(-\min \{p, q\}, \infty)$ if and only if $\lambda \geq \frac{n}{n+1}$.

Theorem 2 in [128] states that the following four statements are equivalent:

1. the function $\frac{\left[\psi^{(n+1)}(x)\right]^{2}}{\psi^{(n)} \psi^{(n+2)(x)}}$ is strictly decreasing from $(0, \infty)$ onto $\left(\frac{n}{n+1}, \frac{n+1}{n+2}\right)$;

2. the sequence $\left\{\left|\frac{\psi^{(n+1)}(x)}{\psi^{(n)}(x)}\right|, x \in(0, \infty)\right\}_{n \in \mathbb{N}}$ is concave;

3. the function $\frac{\left|\psi^{(n)}(x) \psi^{(n+1)}(x)\right|}{\psi^{(n)}(x) \psi^{(n+2)}(x)-\left[\psi^{(n+1)}(x)\right]^{2}}-x$ is strictly decreasing from $(0, \infty)$ onto $\left(-\frac{1}{2}, 0\right)$;

4. the function $\left|\frac{\psi^{(n)}(x)}{\psi^{(n+1)}(x)}\right|$ is convex on $(0, \infty)$. 
We note that the function $h_{\alpha}(t)$ in (3.9) can be regarded as a special case of the function $q_{\alpha, \beta}(t)$ defined by (11.6).

\subsection{A guess on complete monotonicity}

Motivated by Theorem 2.1 in [8] mentioned above, we can consider the function

$$
F_{n, k ; c}(x)=\left[\psi^{(n)}(x)\right]^{2}-c \psi^{(n-k)}(x) \psi^{(n+k)}(x)
$$

for $n>k \geq 1$ on $(0, \infty)$. Similarly, stimulated by Theorems 1 and 2 in [128], we can also discuss the function

$$
\Phi_{n, k, \lambda}(x)=\left[\phi_{n+1}(x)\right]^{2}-\lambda \phi_{n-k+1}(x) \phi_{n+k+1}(x) .
$$

We guess that necessary and sufficient conditions for the functions $\pm F_{n, k ; c}(x)$ to be completely monotonic on $(0, \infty)$ are

$$
c \leq \frac{[(n-1) !]^{2}}{(n-k-1) !(n+k-1) !} \quad \text { and } \quad c \geq \frac{(n !)^{2}}{(n-k) !(n+k) !},
$$

respectively. We will confirm this guess in a subsequent paper soon.

\subsection{Inequalities between polygamma functions}

Theorem 2.2 in [14] states that the double inequality

$$
(n-1) !\left[\frac{\psi^{(k)}(x+1 / 2)}{(-1)^{k-1}(k-1) !}\right]^{n / k}<\left|\psi^{(n)}(x)\right|<(n-1) !\left[\frac{\psi^{(k)}(x)}{(-1)^{k-1}(k-1) !}\right]^{n / k}
$$

holds for $1 \leq k \leq n-1$ and $x>0$. When $n=1$, the left inequality in (3.10) becomes

$$
\psi^{\prime \prime}(x)+\left[\psi^{\prime}\left(x+\frac{1}{2}\right)\right]^{2}<0, \quad x>0
$$

In [33, p. 1008] and [39, p. 110], the double inequality (3.10) was reformulated as

$$
\sqrt[k]{\frac{\left|\psi^{(k)}(x+1 / 2)\right|}{(k-1) !}}<\sqrt[n]{\frac{\left|\psi^{(n)}(x)\right|}{(n-1) !}}<\sqrt[k]{\frac{\left|\psi^{(k)}(x)\right|}{(k-1) !}}
$$

for $x>0$ and $1 \leq k \leq n-1$. The right inequality in (3.11) is equivalent to (3.7). In [33], the left inequality in (3.10) and (3.11) was refined as

$$
\sqrt[n]{\frac{\left|\psi^{(n)}(x)\right|}{(n-1) !}}>\sqrt[k]{\frac{\left|\psi^{(k)}(1 / \ln (1+1 / x))\right|}{(k-1) !}}
$$

for $1 \leq k \leq n-1$ on $(0, \infty)$, since $x<\frac{1}{\ln (1+1 / x)}<x+\frac{1}{2}$ on $(0, \infty)$.

\subsection{Necessary and sufficient conditions}

In [89, Theorem 1 and Corollary 1$]$, the following necessary and sufficient conditions were presented: 
1. the function $\psi^{\prime \prime}(x)+\left[\psi^{\prime}(x+\alpha)\right]^{2}$ is completely monotonic on $(-\min \{0, \alpha\}, \infty)$ if and only if $\alpha \leq 0$;

2. the function

$$
-\left\{\psi^{\prime \prime}(x)+\left[\psi^{\prime}(x+\alpha)\right]^{2}\right\}
$$

is completely monotonic if

$$
\alpha \geq \sup _{x \in(0, \infty)} \frac{x}{\phi^{-1}\left(\left[2(x+1)^{2}-1\right] e^{2 x}\right)},
$$

where $\phi(x)=x \operatorname{coth} x$ for $x \in(0, \infty)$ and $\phi^{-1}$ is the inverse function of $\phi$;

3. for $\alpha \geq \frac{1}{6}$, the function (3.12) is completely monotonic on $(0, \infty)$.

Equivalently speaking, for $\beta \in \mathbb{R}$ and $x>-\min \{0, \beta\}$,

1. the function

$$
\psi^{\prime \prime}(x+\beta)+\left[\psi^{\prime}(x)\right]^{2}
$$

is completely monotonic if and only if $\beta \geq 0$;

2. if

$$
\beta \leq-\sup _{x \in(0, \infty)} \frac{x}{\phi^{-1}\left(\left[2(x+1)^{2}-1\right] e^{2 x}\right)}<0
$$

the negative of (3.14) is completely monotonic;

3. for $\beta \leq-\frac{1}{6}$, the negative of (3.14) is completely monotonic on the interval $(-\min \{0, \beta\}, \infty)$.

\subsection{Complete monotonicity with a parameter}

In [89, Theorem 3], it was found that the function $\left[\psi^{\prime}(x)\right]^{2}+\lambda \psi^{\prime \prime}(x)$ is completely monotonic on $(0, \infty)$ if and only if $\lambda \leq 1$.

\subsection{Uniqueness of complete monotonicity}

In [38, Theorem 1], it was proved that, among the functions

$$
f_{m, n}(x)=\left[\psi^{(m)}(x)\right]^{2}+\psi^{(n)}(x)
$$

on $(0, \infty)$ for $m, n \in \mathbb{N}$, the functions $f_{1,2}(x)$ and $f_{m, 2 n-1}(x)$ are completely monotonic on $(0, \infty)$, but complete monotonicity of $f_{m, 2 n-1}(x)$ is trivial, and the functions $f_{m, 2 n}(x)$ for $(m, n) \neq(1,1)$ are not monotonic and does not keep the same sign on $(0, \infty)$.

\subsection{Completely monotonic degree}

In [47, p. 2273, Corollary 3], among others, it was deduced that the functions $x^{2} \Delta(x)$ and $x^{3} \Delta(x)$ are completely monotonic on $(0, \infty)$. These imply that $\operatorname{deg}_{\mathrm{cm}}^{x}[\Delta(x)] \geq 3$. For more information, please refer to the series of papers [47-49,51].

With the help of [68], it was discovered in [65] that

$$
\operatorname{deg}_{\mathrm{cm}}^{x}[\Delta(x)]=4 .
$$




\subsection{Three $q$-analogs of the third origin}

In [7, pp. 80-81, Lemma 4.6], the inequality (2.6) was generalized as

$$
\Delta_{q}(x)=\left[\psi_{q}^{\prime}(x)\right]^{2}+\psi_{q}^{\prime \prime}(x)>0
$$

for $q>1$ on $(0, \infty)$. In [24, p. 13], it was established that

$$
\left[\psi_{q}^{\prime}(x)\right]^{2}-\frac{q^{x} \ln q}{1-q} \psi_{q}^{\prime \prime}(x) \geq 0, \quad 0<q<1, x>0 .
$$

In [73, Theorem 1.1], it was presented that

1. for $q>1$, the function $\Delta_{q}(x)$ is completely monotonic with respect to $x \in(0, \infty)$;

2. for $0<q<1$, the function

$$
\left[\psi_{q}^{\prime}(x)-\ln q\right]^{2}+\psi_{q}^{\prime \prime}(x)
$$

is completely monotonic with respect to $x \in(0, \infty)$.

\section{Generalizations of the first origin}

The generalizations of the first origin have been developing in recent years.

\subsection{Some basic methods}

In order to present convexity and monotonicity of the function $z_{s, t}(x)$ defined by (2.1), it is enough to show the positivity or negativity of the function $\Delta_{s, t}(x)$. However, it was observed that the function $\Delta_{s, t}(x)$ or its negative should be completely monotonic. In order to verify this observation, there was a barrier to be overcome, the barrier is to significantly simplify and tidy up any high order derivatives of $\Delta_{s, t}(x)$ with respect to $x$. How to deal with high order derivatives of $\Delta_{s, t}(x)$ easily and simply? This problem was nicely and smartly solved in [30, 88]: utilizing the simple but effectual tools stated in Sect. 3.1.

\subsection{Complete monotonicity of divided differences}

In [30, Theorem 1] and [88, Theorem 1.2], it was found that the function $\Delta_{s, t}(x)$, defined by (2.2), for $|t-s|<1$ and $-\Delta_{s, t}(x)$ for $|t-s|>1$ are completely monotonic with respect to $x \in(-\min \{s, t\}, \infty)$. As a consequence, meanwhile, complete monotonicity of $\Delta(x)$ can be recovered once again.

\subsection{Complete monotonicity of divided differences with a parameter}

For $s, t, \lambda \in \mathbb{R}$ and $\alpha=\min \{s, t\}$, the function

$$
\Delta_{s, t ; \lambda}(x)= \begin{cases}{\left[\frac{\psi(x+t)-\psi(x+s)}{t-s}\right]^{2}+\lambda \frac{\psi^{\prime}(x+t)-\psi^{\prime}(x+s)}{t-s},} & s \neq t \\ {\left[\psi^{\prime}(x+s)\right]^{2}+\lambda \psi^{\prime \prime}(x+s),} & s=t .\end{cases}
$$

In [91, Theorem 1.1], complete monotonicity of the function $\Delta_{s, t}(x)$ was generalized as follows:

1. when $0<|t-s|<1$,

(a) the function $\Delta_{s, t ; \lambda}(x)$ is completely monotonic on $(-\alpha, \infty)$ if and only if $\lambda \leq 1$;

(b) the function $-\Delta_{s, t ; \lambda}(x)$ is completely monotonic if and only if $\lambda \geq \frac{1}{|t-s|}$; 
2. when $|t-s|>1$,

(a) the function $\Delta_{s, t ; \lambda}(x)$ is completely monotonic on $(-\alpha, \infty)$ if and only if $\lambda \leq \frac{1}{|t-s|}$;

(b) the function $-\Delta_{s, t ; \lambda}(x)$ is completely monotonic if and only if $\lambda \geq 1$;

3. when $|t-s|=1$,
(a) the function $\Delta_{s, t ; \lambda}(x)$ is completely monotonic if and only if $\lambda<1$;
(b) the function $-\Delta_{s, t ; \lambda}(x)$ is completely monotonic if and only if $\lambda>1$;
(c) and $\Delta_{s, t ; 1}(x) \equiv 0$;

4. when $s=t$, the function $\Delta_{s, s ; \lambda}(x)$ is completely monotonic on $(-s, \infty)$ if and only if $\lambda \leq 1$.

Consequently, [89, Theorem 3], which shows that the function $\left[\psi^{\prime}(x)\right]^{2}+\lambda \psi^{\prime \prime}(x)$ is completely monotonic on $(0, \infty)$ if and only if $\lambda \leq 1$, can be derived again.

\section{Related generalizations}

There are several generalizations related to the above results and conclusions.

\subsection{The difference between the trigamma function and rational functions}

By the formula (3.3), it is easy to see that the function $(-1)^{n+1} \psi^{(n)}(x)$ for $n \in \mathbb{N}$ is completely monotonic on $(0, \infty)$.

The functions

$$
\psi^{\prime}(x)-\frac{1}{x} \quad \text { and } \quad \psi^{\prime}(x)-\frac{1}{x}-\frac{1}{2 x^{2}}=\psi^{\prime}(x)-\frac{2 x+1}{2 x^{2}}
$$

are completely monotonic on $(0, \infty)$. This can be derived from [2, pp. 374-375, Theorem 1], [20, Theorems 1 and 2], [29, Theorem 1.1], [63, Theorem 1.3], [86, Theorem 1] and the closely related references therein. For more general results, please refer to [39, Sect. 1.4] and the closely related references therein.

\subsection{The difference between the trigamma function and a rational function}

It was concluded in [40, Remark 2] that the function

$$
\begin{aligned}
H_{1}(x)= & \psi^{\prime}(x)-\frac{Q_{21}(x)}{1800 x^{2}(1+x)^{10}(2+x)^{10}} \\
= & \psi^{\prime}(x)-\frac{1}{2 x}-\frac{3}{4 x^{2}}-\frac{28}{5(x+1)}+\frac{51}{10(x+2)}+\frac{251}{120(x+1)^{2}} \\
& +\frac{331}{120(x+2)^{2}}-\frac{7}{6(x+1)^{3}}+\frac{17}{12(x+2)^{3}}+\frac{13}{90(x+1)^{4}} \\
& +\frac{49}{72(x+2)^{4}}-\frac{13}{180(x+1)^{5}}+\frac{47}{180(x+2)^{5}}-\frac{1}{120(x+1)^{6}} \\
& -\frac{1}{60(x+2)^{6}}+\frac{1}{180(x+1)^{7}}-\frac{2}{45(x+2)^{7}}+\frac{1}{200(x+1)^{8}} \\
& -\frac{13}{600(x+2)^{8}}+\frac{1}{900(x+1)^{9}}-\frac{1}{450(x+2)^{9}} \\
& -\frac{1}{1800(x+1)^{10}}+\frac{1}{450(x+2)^{10}}
\end{aligned}
$$

is completely monotonic on $(0, \infty)$, where

$$
Q_{21}(x)=1,382,400+21,657,600 x+162,792,960 x^{2}+778,137,600 x^{3}
$$




$$
\begin{aligned}
& +2,645,782,983 x^{4}+6,789,381,590 x^{5}+13,626,443,025 x^{6} \\
& +21,889,330,810 x^{7}+28,579,049,475 x^{8}+30,634,381,522 x^{9} \\
& +27,125,436,630 x^{10}+19,896,883,200 x^{11}+12,088,287,630 x^{12} \\
& +6,063,596,590 x^{13}+2,494,770,300 x^{14}+832,958,400 x^{15} \\
& +222,060,150 x^{16}+46,134,540 x^{17}+7,195,500 x^{18} \\
& +792,300 x^{19}+54,900 x^{20}+1800 x^{21}
\end{aligned}
$$

This result was proved in [40, Theorem 1].

\subsection{Completely monotonic degree is one}

In [32, Theorem 1], complete monotonicity of $H_{1}(x)$ was strengthened as

$$
\operatorname{deg}_{\mathrm{cm}}^{x}\left[H_{1}(x)\right]=1
$$

and the integral representation

$$
H_{1}(x)=\int_{0}^{\infty} q(t) e^{-x t} \mathrm{~d} t
$$

of the form (1.1) or (1.3) was concluded in [32, Remark 2], where

$$
q(t)=\frac{\theta(t)}{653,184,000 e^{2 t}\left(e^{t}-1\right)}
$$

and

$$
\begin{aligned}
\theta(t)= & 163,296,000(t-2) e^{3 t}-e^{2 t}\left(t^{9}-18 t^{8}-648 t^{7}-5040 t^{6}\right. \\
& +45,360 t^{5}+1,965,600 t^{4}-15,724,800 t^{3}+381,024,000 t^{2} \\
& -1,856,131,200 t+3,331,238,400)+e^{t}\left(5 t^{9}-54 t^{8}-3456 t^{7}\right. \\
& -45,360 t^{6}-45,360 t^{5}+9,072,000 t^{4}+58,363,200 t^{3} \\
& \left.+843,696,000 t^{2}+435,456,000 t+6,989,068,800\right)-4\left(t^{9}-9 t^{8}\right. \\
& -702 t^{7}-10,080 t^{6}-22,680 t^{5}+1,776,600 t^{4}+18,522,000 t^{3} \\
& \left.+115,668,000 t^{2}+450,424,800 t+832,809,600\right)
\end{aligned}
$$

with $q(0)=0, q(t) \geq 0$, and $q^{\prime}(t)>0$ on $(0, \infty)$.

\subsection{A difference between the trigamma function and a rational function}

In [136, Remark 1], it was concluded that the functions

$$
\begin{aligned}
H_{2}(x) & =\psi^{\prime}(x)-\frac{Q_{7}(x)}{6 x^{2}(1+x)^{4}(2+x)^{2}} \\
& =\psi^{\prime}(x)-\frac{24+120 x+283 x^{2}+399 x^{3}+345 x^{4}+181 x^{5}+51 x^{6}+6 x^{7}}{6 x^{2}(1+x)^{4}(2+x)^{2}}
\end{aligned}
$$




$$
\begin{aligned}
= & \psi^{\prime}(x)-\left[\frac{1}{x^{2}}+\frac{43}{6(x+1)}-\frac{7}{2(x+1)^{2}}+\frac{2}{(x+1)^{3}}\right. \\
& \left.-\frac{1}{2(x+1)^{4}}-\frac{37}{6(x+2)}-\frac{13}{6(x+2)^{2}}\right]
\end{aligned}
$$

and

$$
\begin{aligned}
H_{3}(x)= & \frac{Q_{6}(x)}{24 x^{2}(x+1)^{4}(x+2)}-\psi^{\prime}(x) \\
= & \frac{24 x^{6}+156 x^{5}+412 x^{4}+621 x^{3}+523 x^{2}+242 x+48}{24 x^{2}(x+1)^{4}(x+2)}-\psi^{\prime}(x) \\
= & \frac{1}{x^{2}}+\frac{13}{24 x}+\frac{55}{24(x+1)}-\frac{15}{8(x+1)^{2}}+\frac{35}{24(x+1)^{3}} \\
& -\frac{1}{2(x+1)^{4}}-\frac{11}{6(x+2)}-\psi^{\prime}(x)
\end{aligned}
$$

are completely monotonic on $(0, \infty)$. Consequently, as claimed in [136, Remark 2], integrating the inequalities $H_{2}(x)>0$ and $H_{3}(x)>0$ yields a double inequality,

$$
\begin{aligned}
& \frac{42 t^{3}+t^{2}-81 t-48}{48 t(t+1)^{3}}+\frac{13 \ln t+55 \ln (t+1)-44 \ln (t+2)}{24} \\
& <\psi(t)<\frac{28 t^{4}+87 t^{3}+73 t^{2}+3 t-12}{6 t(t+1)^{3}(t+2)}+\frac{43 \ln (t+1)-37 \ln (t+2)}{6}, \quad t>0 .
\end{aligned}
$$

Can one compute completely monotonic degrees of the functions $H_{2}(x)$ and $H_{3}(x)$ on $(0, \infty)$, as one did in [32]?

\subsection{Necessary and sufficient conditions}

In [72, Remark 3], it was deduced that the function

$$
\psi^{\prime}(x)-\frac{\lambda\left(4+8 x+5 x^{2}\right)}{24 x(1+x)^{3}(2+x)^{2}}-\frac{Q_{7}(x)}{6 x^{2}(1+x)^{4}(2+x)^{2}}
$$

is completely monotonic on $(0, \infty)$ if and only if $\lambda \leq 0$, and its negative is completely monotonic on $(0, \infty)$ if and only if $\lambda \geq 4$.

What are completely monotonic degrees of the function (5.2) for $\lambda \leq 0$ and its negative for $\lambda \geq 4$ on $(0, \infty)$ ?

\subsection{A difference between the trigamma function and a rational function of a parameter}

Let $\alpha \in \mathbb{R}$ and $x>-\min \{0, \alpha\}$. It was proved in [89, Theorem 2 and Corollary 1$]$ that

1. the function

$$
\psi^{\prime}(x+\alpha)-\frac{1}{2(x+\alpha)^{2}}-\frac{(x+\alpha)^{2}}{x^{3}}
$$

is completely monotonic if and only if $\alpha \leq 0$;

2. the negative of the function (5.3) is completely monotonic if the inequality (3.13) is valid; 
3. the necessary condition for the negative of (5.3) to be completely monotonic on $x \in(0, \infty)$ is

$$
\alpha \geq \sup _{x \in(0, \infty)}\left\{x\left[1-\sqrt[3]{\frac{2 x}{2 x^{2} \psi^{\prime}(x)-1}}\right]\right\}
$$

4. for $\alpha \geq \frac{1}{6}$, the negative of (5.3) is completely monotonic on $(0, \infty)$; equivalently speaking, for $\beta \leq-\frac{1}{6}$, the function $\frac{1}{2 x^{2}}+\frac{x^{2}}{(x+\beta)^{3}}-\psi^{\prime}(x)$ is completely monotonic on $(-\min \{0, \beta\}, \infty)$.

We guess that the conditions in (3.13) and (5.4) should be necessary and sufficient.

\subsection{A difference between a divided difference of and a rational function}

As by-product of [62, p. 524, Theorem 1.1] and [88, p. 1978, Theorem 1.1], the following complete monotonicity for the divided difference form of the second function in (5.1) was acquired: the function

$$
\delta_{s, t}(x)= \begin{cases}\frac{\psi(x+t)-\psi(x+s)}{t-s}-\frac{2 x+s+t+1}{2(x+s)(x+t)}, & s \neq t, \\ \psi^{\prime}(x+s)-\frac{1}{x+s}-\frac{1}{2(x+s)^{2}}, & s=t,\end{cases}
$$

for $|t-s|<1$ and $-\delta_{s, t}(x)$ for $|t-s|>1$ are completely monotonic on $x \in(-\alpha, \infty)$, where $s$ and $t$ are real numbers and $\alpha=\min \{s, t\}$.

\subsection{A difference of a divided difference of and a rational function with a parameter}

In [91, Theorem 1.3], along with generalizing $\Delta_{s, t}(x)$ to $\Delta_{s, t ; \lambda}(x)$ in (4.1), the authors generalized the function $\delta_{s, t}(x)$ as

$$
\theta_{s, t ; \lambda}(x)= \begin{cases}\frac{\psi(x+t)-\psi(x+s)}{t-s}-\frac{1+\lambda(2 x+s+t)}{2(x+s)(x+t)}, & s \neq t, \\ \psi^{\prime}(x+s)-\frac{\lambda}{x+s}-\frac{1}{2(x+s)^{2}}, & s=t,\end{cases}
$$

on $(-\min \{s, t\}, \infty)$ for $s, t, \lambda \in \mathbb{R}$, and presented the following complete monotonicity:

1. when $0<|t-s|<1$,
(a) the function $\theta_{s, t ; \lambda}(x)$ is completely monotonic if and only if $\lambda \leq 1$;
(b) the function $-\theta_{s, t ; \lambda}(x)$ is completely monotonic if and only if $\lambda \geq \frac{1}{|t-s|}$;

2. when $|t-s|>1$,
(a) the function $\theta_{s, t ; \lambda}(x)$ is completely monotonic if and only if $\lambda \leq \frac{1}{|t-s|}$;
(b) the function $-\theta_{s, t ; \lambda}(x)$ is completely monotonic if and only if $\lambda \geq 1$;

3. when $|t-s|=1$,

(a) the function $\theta_{s, t ; \lambda}(x)$ is completely monotonic if and only if $\lambda<1$;

(b) the function $-\theta_{s, t ; \lambda}(x)$ is completely monotonic if and only if $\lambda>1$;

(c) and $\theta_{s, t ; 1}(x) \equiv 0$;

4. when $s=t$, the function $\theta_{s, s ; \lambda}(x)$ is completely monotonic if and only if $\lambda \leq 1$. 


\subsection{Necessary and sufficient conditions of complete monotonicity}

In $[83,108]$, the authors alternatively generalized the function $\delta_{s, t}(x)$ in $(5.5)$ as

$$
\delta_{s, t ; \lambda}(x)= \begin{cases}\frac{\psi(x+t)-\psi(x+s)}{t-s}-\frac{2 x+s+t+2 \lambda}{2(x+s)(x+t)}, & s \neq t, \\ \psi^{\prime}(x+s)-\frac{1}{x+s}-\frac{\lambda}{(x+s)^{2}}, & s=t,\end{cases}
$$

on $(-\alpha, \infty)$ for $s, t, \lambda \in \mathbb{R}$ and $\alpha=\min \{s, t\}$, and discovered the following necessary and sufficient conditions of complete monotonicity:

1. when $|t-s|<1$,

(a) the function $\delta_{s, t ; \lambda}(x)$ is completely monotonic on $(-\alpha, \infty)$ if and only if $\lambda \leq \frac{1}{2}$;

(b) the function $-\delta_{s, t ; \lambda}(x)$ is completely monotonic on $(-\alpha, \infty)$ if and only if $\lambda \geq 1-\frac{|t-s|}{2}$

2. when $|t-s|>1$,

(a) the function $\delta_{s, t ; \lambda}(x)$ is completely monotonic on $(-\alpha, \infty)$ if and only if $\lambda \leq 1-\frac{|t-s|}{2}$

(b) the function $-\delta_{s, t ; \lambda}(x)$ is completely monotonic on $(-\alpha, \infty)$ if and only if $\lambda \geq \frac{1}{2}$;

3. when $|t-s|=1$,

(a) the function $\delta_{s, t ; \lambda}(x)$ is completely monotonic on $(-\alpha, \infty)$ if and only if $\lambda<\frac{1}{2}$;

(b) the function $-\delta_{s, t ; \lambda}(x)$ is completely monotonic on $(-\alpha, \infty)$ if and only if $\lambda>\frac{1}{2}$;

(c) the function $\delta_{s, t ; \lambda}(x)$ is identically zero if and only if $\lambda=\frac{1}{2}$.

\subsection{Complete monotonicity of $q$-analogs}

In $[67,105,135]$, the second function in $(5.1)$ was generalized to the $q$-analogs

$$
f_{q}(x)=\psi_{q}^{\prime}(x)-\frac{(1-q) q^{x}}{1-q^{x}}-\frac{1}{2}\left[\frac{(1-q) q^{x}}{1-q^{x}}\right]^{2}
$$

and

$$
F_{q}(x)=\psi_{q}^{\prime}(x)-\frac{1-q}{1-q^{x}}-\frac{1}{2}\left(\frac{1-q}{1-q^{x}}\right)^{2}+\frac{1}{2}(1-q)(3-q)
$$

on $(0, \infty)$ for $0<q<1$ and these two $q$-analogs were proved to be completely monotonic on $(0, \infty)$.

For $0<q<1$, we guess that the functions

$$
\begin{aligned}
I_{q}(x)= & \psi_{q}^{\prime}(x)-\frac{1-q}{1-q^{x}}-\frac{1}{2}\left(\frac{1-q}{1-q^{x}}\right)^{2}-\frac{1}{6}\left(\frac{1-q}{1-q^{x}}\right)^{3} \\
& +\frac{1}{30}\left(\frac{1-q}{1-q^{x}}\right)^{5}+\frac{1-q}{30}\left(49-21 q-q^{2}+4 q^{3}-q^{4}\right)
\end{aligned}
$$

and

$$
\begin{aligned}
J_{q}(x)= & \psi_{q}^{\prime}(x)-\frac{1-q}{1-q^{x}}-\frac{1}{2}\left(\frac{1-q}{1-q^{x}}\right)^{2}-\frac{1}{6}\left(\frac{1-q}{1-q^{x}}\right)^{2} \\
& +\frac{1}{30}\left[\frac{(1-q) q^{x}}{1-q^{x}}\right]^{5}+\frac{1-q}{6}\left(10-5 q+q^{2}\right)
\end{aligned}
$$

are completely monotonic with respect to $x \in(0, \infty)$. 


\section{Applications}

Some of the above results and conclusions have been applied to find inequalities, monotonicity, logarithmically completely monotonicity of some functions involving the gamma function $\Gamma(x)$, the ratio $\frac{\Gamma(x+a)}{\Gamma(x+b)}$, polygamma functions $\psi^{(k)}(x)$ for $k \geq 0$, and so on.

\subsection{Monotonicity and convexity}

In [34, Theorem 2], by virtue of the inequality (2.6), it was proved that the function

$$
e^{\psi(x+1)}-x
$$

is strictly decreasing and strictly convex on $(-1, \infty)$.

In [123], Theorems 1 and 2 state that

1. the function

$$
\exp \left[\psi\left(x+\frac{1}{2}\right)-\frac{1}{24} \frac{1}{x^{2}+7 / 40}\right]-x
$$

is decreasing from $\left(-\frac{1}{2}, \infty\right)$ onto $\left(0, \frac{1}{2}\right)$ and convex on $\left(-\frac{1}{2}, \infty\right)$;

2. the function

$$
\exp \left[\psi\left(x+\frac{1}{2}\right)\right]-x \exp \left(\frac{1}{24} \frac{1}{x^{2}+7 / 40}\right)
$$

is decreasing from $(0, \infty)$ onto $\left(0, \frac{1}{4 e^{\gamma}}\right)$ and convex on $\left(\frac{1}{2}, \infty\right)$, where $\gamma=0.57721566 \ldots$ is the Euler-Mascheroni constant.

\subsection{Alternative proofs of the first origin}

By virtue of the equality (2.2), we see readily that complete monotonicity of the function $\Delta_{s, t}(x)$ implies the convexity, and then the monotonicity, of the function $z_{s, t}(x)$ defined by (2.1). Therefore, alternative proofs of [23, Theorem 1] were provided in [19, 30, 31, 88, 99] and the closely related references therein.

\subsection{A generalization of the first origin}

As an application of [91, Theorem 1.1] and as a generalization of [23, Theorem 1, Corollaries 2 and 3], [34, Theorem 2], and [89, p. 75, Corollary 2], Theorem 1.5 in [91] states that the function

$$
z_{s, t ; \lambda}(x)= \begin{cases}{\left[\frac{\Gamma(x+t)}{\Gamma(x+s)}\right]^{1 /[\lambda(t-s)]},} & s \neq t, \\ e^{\psi(x+s) / \lambda}, & s=t,\end{cases}
$$

for $s, t \in \mathbb{R}, \alpha=\min \{s, t\}$, and $\lambda \neq 0$ has the following properties:

1. when $0<|t-s|<1$,

(a) the function $z_{s, t ; \lambda}(x)$ is convex on $(-\alpha, \infty)$ if and only if $\lambda \leq 1$,

(b) the function $z_{s, t ; \lambda}(x)$ is concave on $(-\alpha, \infty)$ if and only if $\lambda \geq \frac{1}{|t-s|}$;

2. when $|t-s|>1$,
(a) the function $z_{s, t ; \lambda}(x)$ is convex on $(-\alpha, \infty)$ if and only if $\lambda \leq \frac{1}{|t-s|}$,

(b) the function $z_{s, t ; \lambda}(x)$ is concave on $(-\alpha, \infty)$ if and only if $\lambda \geq 1$; 
3. when $s=t$, the function $z_{s, s ; \lambda}(x)$ is convex on $(-s, \infty)$ if and only if $\lambda \leq 1$;

4. when $|t-s|=1$,
(a) the function $z_{s, t ; \lambda}(x)$ is convex on $(-\alpha, \infty)$ if and only if $\lambda<1$,
(b) the function $z_{s, t ; \lambda}(x)$ is concave on $(-\alpha, \infty)$ if and only if $\lambda>1$,
(c) $z_{s, t ; 1}(x)=x+\alpha$ on $(-\alpha, \infty)$.

\subsection{Monotonicity and inequalities for $q$-analogs}

Using complete monotonicity of the function $\Delta_{q}(x)$ on $(0, \infty)$, the following monotonicity cases and inequalities were established in [73, Corollary 1.1]: the functions

$$
\begin{aligned}
& \phi_{q}(x)= \begin{cases}\psi_{q}(x)+\ln \left[\exp \frac{(\ln q) q^{x}}{q^{x}-1}-1\right], & q>1, \\
\psi(x)+\ln \left(e^{1 / x}-1\right), & q=1,\end{cases} \\
& \varphi_{q}(x)= \begin{cases}\psi_{q}(x)+\ln \left(\exp \frac{\ln q}{q^{x}-1}-1\right), & q>1, \\
\psi(x)+\ln \left(e^{1 / x}-1\right), & q=1,\end{cases} \\
& \Phi_{q}(x)= \begin{cases}\psi_{q}(x)-(\ln q) x+\ln \left[\exp \frac{(\ln q) q^{x}}{q^{x}-1}-1\right], & 0<q<1, \\
\psi(x)+\ln \left(e^{1 / x}-1\right), & q=1,\end{cases} \\
& \Theta_{q}(x)= \begin{cases}\psi_{q}(x)-(\ln q) x+\ln \left(\exp \frac{\ln q}{q^{x}-1}-1\right), & 0<q<1, \\
\psi(x)+\ln \left(e^{1 / x}-1\right), & q=1,\end{cases}
\end{aligned}
$$

are strictly increasing on $(0, \infty)$; consequently,

1. the double inequality

$$
a-\ln \left(e^{1 / x}-1\right)<\psi(x)<b-\ln \left(e^{1 / x}-1\right)
$$

holds on $(0, \infty)$ if and only if $a \leq-\gamma=-0.577 \ldots$ and $b \geq 0$;

2. for $0<q<1$, the double inequality

$$
\begin{aligned}
& \psi_{q}(1)+(\ln q) x-\ln \left[\exp \frac{(\ln q) q^{x}}{q^{x}-1}-1\right] \\
& <\psi_{q}(x)<\ln \frac{\ln q}{q-1}+(\ln q) x-\ln \left[\exp \frac{(\ln q) q^{x}}{q^{x}-1}-1\right]
\end{aligned}
$$

holds on $(0, \infty)$, where the constants $\psi_{q}(1)$ and $\ln \left(\frac{\ln q}{q-1}\right)$ are the best possible;

3 . for $q>1$, the double inequality

$$
\begin{aligned}
& \psi_{q}(1)-\ln q-\ln \left(\exp \frac{\ln q}{q^{x}-1}-1\right) \\
& <\psi_{q}(x)<\ln \frac{\ln q}{q-1}-\frac{\ln q}{2}-\ln \left(\exp \frac{\ln q}{q^{x}-1}-1\right)
\end{aligned}
$$

holds on $(0, \infty)$, where the scalars $\psi_{q}(1)-\ln q$ and $\ln \frac{\ln q}{q-1}-\frac{\ln q}{2}$ are the best possible. For properties of the function $\psi(x)+\ln \left(e^{1 / x}-1\right)$ and related functions, please refer to [ 4 , $13,14,28,53]$ and the closely related references therein. 


\subsection{Double inequalities for the gamma function and its $q$-analog}

The inequality (2.6) was applied in [12, Theorem 2.1$]$ to provide a double inequality,

$$
\Gamma\left(x^{*}\right) \exp \left\{\alpha\left[e^{\psi(x)}(\psi(x)-1)+1\right]\right\} \leq \Gamma(x) \leq \Gamma\left(x^{*}\right) \exp \left\{\beta\left[e^{\psi(x)}(\psi(x)-1)+1\right]\right\}
$$

for $x>x^{*}$, where $\alpha=1, \beta=\frac{6 e^{\gamma}}{\pi^{2}}=1.0827 \ldots$, and $x^{*}=1.4616 \ldots$ is the only positive zero of $\psi(x)$ such that $\Gamma\left(x^{*}\right)=0.8856 \ldots$

In [7, Theorem 4.3], the inequality (6.2) was proved to be valid for $x \in(a, b)$ and with the best possible constants

$$
\alpha=\left\{\begin{array}{ll}
Q(b), & \text { if } b<\infty, \\
1, & \text { if } b=\infty,
\end{array} \text { and } \quad \beta=Q(a)\right.
$$

where $0<a<b \leq \infty$ and

$$
Q(x)= \begin{cases}\frac{\ln \Gamma(x)-\ln \Gamma\left(x^{*}\right)}{[\psi(x)-1] e^{\psi(x)+1},} & x \neq x^{*} \\ \frac{1}{\psi^{\prime}\left(x^{*}\right)}, & x=x^{*}\end{cases}
$$

In [7, Theorem 4.8], by establishing and utilizing the inequality (3.17), the inequality (6.2) was generalized to the $q$-analog

$$
\Gamma_{q}\left(x_{0}\right) e^{\alpha\left[e^{\psi_{q}(x)}\left(\psi_{q}(x)-1\right)+1\right]} \leq \Gamma_{q}(x) \leq \Gamma_{q}\left(x_{0}\right) e^{\beta\left[e^{\psi_{q}(x)}\left(\psi_{q}(x)-1\right)+1\right]}
$$

for $x \in(a, b)$ and with the best possible constants

$$
\alpha=\left\{\begin{array}{ll}
Q_{q}(b), & \text { if } b<\infty, \\
0, & \text { if } b=\infty,
\end{array} \text { and } \beta=Q_{q}(a),\right.
$$

where $q>1,0<a<b \leq \infty, x_{0}$ is the only zero of $\psi_{q}(x)$ on $(0, \infty)$, and

$$
Q_{q}(x)= \begin{cases}\frac{\ln \Gamma_{q}(x)-\ln \Gamma_{q}\left(x_{0}\right)}{\left[\psi_{q}(x)-1\right] e^{\psi_{q}(x)}+1}, & x \neq x_{0} ; \\ \frac{1}{\psi_{q}^{\prime}\left(x_{0}\right)}, & x=x_{0} .\end{cases}
$$

\subsection{A monotonicity result}

In [30, Theorem 2], complete monotonicity of the function $\Delta_{s, t}(x)$ defined in (2.2) was applied to generalize the inequality (6.2) to a monotonicity result below. For real numbers $s$ and $t, \alpha=\min \{s, t\}$, and $c \in(-\alpha, \infty)$, let

$$
g_{s, t}(x)= \begin{cases}\frac{1}{t-s} \int_{c}^{x} \ln \left[\frac{\Gamma(u+t)}{\Gamma(u+s)} \frac{\Gamma(c+s)}{\Gamma(c+t)}\right] \mathrm{d} u, & s \neq t, \\ \int_{c}^{x}[\psi(u+s)-\psi(c+s)] \mathrm{d} u, & s=t\end{cases}
$$

on $x \in(-\alpha, \infty)$. Then the function

$$
f_{s, t}(x)= \begin{cases}\frac{g_{s, t}(x)}{\left[g_{s, t}^{\prime}(x)-1\right] \exp \left[g_{s, t}^{\prime}(x)\right]+1}, & x \neq c, \\ \frac{1}{g_{s, t}^{\prime \prime}(c)}, & x=c,\end{cases}
$$

on $x \in(-\alpha, \infty)$ is decreasing for $|t-s|<1$ and increasing for $|t-s|>1$. 


\subsection{Monotonicity and convexity}

In $\left[89\right.$, p. 75 , Corollary 2], complete monotonicity of $\left[\psi^{\prime}(x)\right]^{2}+\lambda \psi^{\prime \prime}(x)$ was utilized to discuss the monotonicity and convexity of the function

$$
f_{p, q}(x)=e^{p \psi(x+1)}-q x
$$

which is a generalization of the function in (6.1), with respect to $x \in(-1, \infty)$ for $p \neq 0$ and $q \in \mathbb{R}$ : the function $f_{p, q}(x)$

1. is strictly decreasing if and only if $p=1$ and $q \geq 1$ or $p<0$ and $q \geq 0$;

2. is strictly increasing if and only if $p=1$ and $q \leq 0$ or $p>1$ and $q \leq 0$;

3. is strictly convex if and only if $p \geq 1$ or $p<0$;

4. has a unique minimum if and only if $p \geq 1$ and $0<q<1$ or $p<0$ and $q<0$.

These results extend and generalize [23, pp. 241-242, Corollary 2].

\subsection{Logarithmically complete monotonicity related to the ratio of gamma functions}

In [88, p. 1980, Theorem 1.4], complete monotonicity of the function $\delta_{s, t}(x)$ defined by (5.5) was applied to derive the following logarithmically complete monotonicity of a function involving the ratio of two gamma functions: for $s, t \in \mathbb{R}$ and $\alpha=\min \{s, t\}$,

1. when $|t-s|>1$, the function

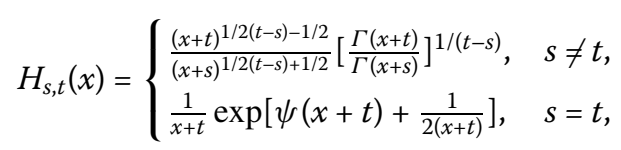

is logarithmically completely monotonic on $(-\alpha, \infty)$;

2. when $|t-s|<1$, the function $\frac{1}{H_{s, t}(x)}$ is logarithmically completely monotonic on $(-\alpha, \infty)$;

3. when $|t-s|=1$, the function $H_{s, t}(x)$ identically equals 1 on $(-\alpha, \infty)$;

4. when $|t-s|<1$, the inequality

$$
\left[\frac{\Gamma(x+t)}{\Gamma(x+s)}\right]^{1 /(t-s)}<\frac{(x+s)^{1 / 2(t-s)+1 / 2}}{(x+t)^{1 / 2(t-s)-1 / 2}}
$$

holds on $(-\alpha, \infty)$;

5. when $|t-s|>1$, the inequality (6.3) is reversed on $(-\alpha, \infty)$.

\subsection{Logarithmically complete monotonicity involving the ratio of gamma functions}

By applying complete monotonicity of the function $\theta_{s, t ; \lambda}(x)$ defined in (5.6), the following results were discovered in [91, Theorem 1.2]. For $s, t, \lambda \in \mathbb{R}$ and $\alpha=\min \{s, t\}$, let

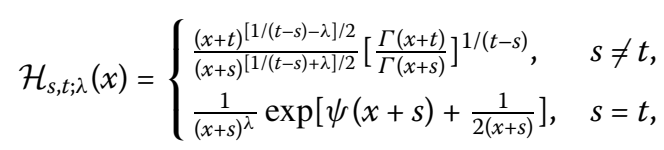

on $(-\alpha, \infty)$. Then 
1. when $0<|t-s|<1$,

(a) the function $\mathcal{H}_{s, t ; \lambda}(x)$ is logarithmically completely monotonic if and only if $\lambda \geq \frac{1}{|t-s|}$

(b) the function $\frac{1}{\mathcal{H}_{s, t ; \lambda}(x)}$ is logarithmically completely monotonic if and only if $\lambda \leq 1$;

2. when $|t-s|>1$,

(a) the function $\mathcal{H}_{s, t ; \lambda}(x)$ is logarithmically completely monotonic if and only if $\lambda \geq 1$;

(b) the function $\frac{1}{\mathcal{H}_{s, t ; \lambda}(x)}$ is logarithmically completely monotonic if and only if $\lambda \leq \frac{1}{|t-s|}$

3. when $s=t$, the function $\frac{1}{\mathcal{H}_{s, s ; \lambda}(x)}$ is logarithmically completely monotonic if and only if $\lambda \leq 1$

4. when $|t-s|=1$,
(a) the function $\mathcal{H}_{s, t ; \lambda}(x)$ is logarithmically completely monotonic if and only if $\lambda>1$;
(b) the function $\frac{1}{\mathcal{H}_{s, s ; \lambda}(x)}$ is logarithmically completely monotonic if and only if $\lambda<1$;
(c) and $\mathcal{H}_{s, t ; 1}(x) \equiv 1$.

\subsection{Logarithmically complete monotonicity concerning the ratio of gamma functions}

By applying complete monotonicity of the function $\delta_{s, t ; \lambda}(x)$ defined in (5.7), the following logarithmically complete monotonicity were obtained in [83, Theorem 1.2] and [108, Theorem 1.3]. For $s, t, \lambda \in \mathbb{R}$ and $\alpha=\min \{s, t\}$, define

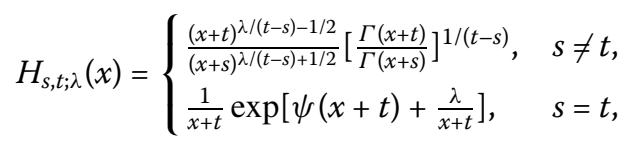

on $(-\alpha, \infty)$. Then

1. when $|t-s|<1$,

(a) the function $H_{s, t ; \lambda}(x)$ is logarithmically completely monotonic if and only if $\lambda \geq 1-\frac{|t-s|}{2}$

(b) the function $\frac{1}{H_{s, t ; \lambda}(x)}$ is logarithmically completely monotonic if and only if $\lambda \leq \frac{1}{2}$;

2. when $|t-s|>1$,

(a) the function $H_{s, t ; \lambda}(x)$ is logarithmically completely monotonic if and only if $\lambda \geq \frac{1}{2}$;

(b) the function $\frac{1}{H_{s, t ; \lambda}(x)}$ is logarithmically completely monotonic if and only if $\lambda \leq 1-\frac{|t-s|}{2} ;$

3. when $|t-s|=1$,

(a) the function $H_{s, t ; \lambda}(x)$ is logarithmically completely monotonic if and only if $\lambda>\frac{1}{2}$;

(b) the function $\frac{1}{H_{s, t ; \lambda}(x)}$ is logarithmically completely monotonic if and only if $\lambda<\frac{1}{2}$;

(c) the function $H_{s, t ; \lambda}(x)$ identically equals 1 on $(-\alpha, \infty)$ if and only if $\lambda=\frac{1}{2}$.

\section{Equivalent inequalities of complete monotonicity}

It is very surprising that some (logarithmically) complete monotonicity cases discussed above are equivalent to some inequalities for sums.

\subsection{First equivalence}

Theorem 1.2 in [62, p. 526] shows that, for $k \geq 0$ and $\theta>0$, 
1. if $a>0$ and $b>0$, then

$$
\sum_{i=0}^{k} \frac{1}{(a+\theta)^{i+1}(b+\theta)^{k-i+1}}+\sum_{i=0}^{k} \frac{1}{a^{i+1} b^{k-i+1}}>2 \sum_{i=0}^{k} \frac{1}{(a+\theta)^{i+1} b^{k-i+1}}
$$

holds for $b-a>-\theta$ and reverses for $b-a<-\theta$;

2. if $a<-\theta$ and $b<-\theta$, then inequalities

$$
\begin{aligned}
& \sum_{i=0}^{2 k} \frac{1}{(a+\theta)^{i+1}(b+\theta)^{2 k-i+1}}+\sum_{i=0}^{2 k} \frac{1}{a^{i+1} b^{2 k-i+1}} \\
& \quad>2 \sum_{i=0}^{2 k} \frac{1}{(a+\theta)^{i+1} b^{2 k-i+1}}
\end{aligned}
$$

and

$$
\begin{aligned}
& \sum_{i=0}^{2 k+1} \frac{1}{(a+\theta)^{i+1}(b+\theta)^{2 k-i+2}}+\sum_{i=0}^{2 k+1} \frac{1}{a^{i+1} b^{2 k-i+2}} \\
& \quad<2 \sum_{i=0}^{2 k+1} \frac{1}{(a+\theta)^{i+1} b^{2 k-i+2}}
\end{aligned}
$$

hold for $b-a>-\theta$ and reverse for $b-a<-\theta$;

3. if $-\theta<a<0$ and $-\theta<b<0$, then inequality (7.2) holds and inequality (7.3) is valid for $a+b+\theta>0$ and is reversed for $a+b+\theta<0$;

4. if $a<-\theta$ and $b>0$, then inequality (7.2) holds and inequality (7.3) is valid for $a+b+\theta>0$ and is reversed for $a+b+\theta<0$;

5. if $a>0$ and $b<-\theta$, then inequality (7.2) is reversed and inequality (7.3) holds for $a+b+\theta<0$ and reverses for $a+b+\theta>0$

6. if $b=a-\theta$, then inequalities (7.1), (7.2) and (7.3) become equalities.

Theorem 1.3 in [62, p. 526] states that the inequality (7.1) for $a, b>0$ is equivalent to [62, p. 524, Theorem 1.1] and [88, p. 1978, Theorem 1.1] mentioned in Sect. 5.7 in this paper.

\subsection{Second equivalence}

The (logarithmically) complete monotonicity of the functions $\delta_{s, t ; \lambda}(x)$ and $H_{s, t ; \lambda}(x)$ are equivalent to the following inequality (7.4) for the case $a, b>0$. See [108, Remarks 1.8 and 1.9].

Theorem 1.7 in [108], which is a generalization of [62, Theorem 1.2] mentioned above, states that, for $k \in \mathbb{N}, \theta>0$, and $a, b, \lambda \in \mathbb{R}$,

1. the inequality

$$
\begin{aligned}
\sum_{i=0}^{k} \frac{1}{(a+\theta)^{i+1} b^{k-i+1}}< & \frac{2 \theta(1-\lambda)-b+a}{2(\theta-b+a)} \sum_{i=0}^{k} \frac{1}{a^{i+1} b^{k-i+1}} \\
& +\frac{2 \theta \lambda-b+a}{2(\theta-b+a)} \sum_{i=0}^{k} \frac{1}{(a+\theta)^{i+1}(b+\theta)^{k-i+1}}
\end{aligned}
$$

holds 
(a) if $a, b>0$ and

(1) either $0<|b-a|<\theta$ and $\lambda \leq \frac{1}{2}$,

(2) or $b-a<-\theta$ and $\lambda \leq 1+\frac{b-a}{2 \theta}$,

(3) or $b-a>\theta$ and $\lambda \geq \frac{1}{2}$;

(b) if $a, b<-\theta$,

(1) $k$ is even and

$$
\left\{\begin{array}{l}
\text { either } 0<|b-a|<\theta \text { and } \lambda \geq \frac{1}{2}, \\
\text { or } a-b<-\theta \text { and } \lambda \geq \frac{b-a}{2 \theta}, \\
\text { or } a-b>\theta \text { and } \lambda \leq \frac{1}{2}
\end{array}\right.
$$

(2) $k$ is odd and

$$
\left\{\begin{array}{l}
\text { either } 0<|b-a|<\theta \text { and } \lambda \leq \frac{|b-a|}{2 \theta}, \\
\text { or } a-b<-\theta \text { and } \lambda \leq \frac{1}{2} \\
\text { or } a-b>\theta \text { and } \lambda \geq \frac{a-b}{2 \theta}
\end{array}\right.
$$

(c) if $-\theta<a<0,-\theta<b<0$ and

(1) $k$ is even and $0<\lambda \leq 1$,

(2) $k$ is odd,

$$
\left\{\begin{array}{l}
\text { either }-2 \theta<a+b<-\theta \text { and } \lambda \geq \frac{1}{2} \\
\text { or } a+b+\theta=0 \text { and } \lambda>\frac{1}{2}
\end{array}\right.
$$

(d) if either $a<-\theta$ and $b>0$ or $a>0$ and $b<-\theta$,

(1) $k$ is even and $0 \leq \lambda \leq 1$,

(2) $k$ is odd and

$$
\left\{\begin{array}{l}
\text { either } a+b+\theta<0 \text { and } \lambda \leq \frac{1}{2} \\
\text { or } a+b+\theta=0 \text { and } \lambda<\frac{1}{2}
\end{array}\right.
$$

2. the inequality (7.1) is reversed

(a) if $a>0, b>0$ and
(1) either $0<|b-a|<\theta$ and $\lambda \geq 1-\frac{|b-a|}{2 \theta}$,
(2) or $b-a<-\theta$ and $\lambda \geq \frac{1}{2}$,
(3) or $b-a>\theta$ and $\lambda \leq 1-\frac{b-a}{2 \theta}$;

(b) if $a<-\theta$ and $b<-\theta$,

(1) $k$ is even and

$$
\left\{\begin{array}{l}
\text { either } 0<|b-a|<\theta \text { and } \lambda \leq \frac{|b-a|}{2 \theta}, \\
\text { or } a-b<-\theta \text { and } \lambda \leq \frac{1}{2} \\
\text { or } a-b>\theta \text { and } \lambda \geq \frac{a-b}{2 \theta}
\end{array}\right.
$$


(2) $k$ is odd and

$$
\left\{\begin{array}{l}
\text { either } 0<|b-a|<\theta \text { and } \lambda \geq \frac{1}{2} \\
\text { or } a-b<-\theta \text { and } \lambda \geq \frac{b-a}{2 \theta} \\
\text { or } a-b>\theta \text { and } \lambda \leq \frac{1}{2}
\end{array}\right.
$$

(c) if $-\theta<a<0,-\theta<b<0, k$ is odd and

(1) either $0>a+b>-\theta$ and $\lambda \leq \frac{1}{2}$,

(2) or $a+b+\theta=0$ and $\lambda<\frac{1}{2}$;

(d) if either $a<-\theta$ and $b>0$ or $a>0$ and $b<-\theta$, $k$ is odd and

(1) either $a+b+\theta>0$ and $\lambda \geq \frac{1}{2}$,

(2) or $a+b+\theta=0$ and $\lambda>\frac{1}{2}$.

3. the sign $<$ in (7.1) can be replaced by the equal sign $=$ if $a+b+\theta=0, \lambda=\frac{1}{2}$ and
(a) either $-1<a<0$ and $-1<b<0$
(b) or $a<-1$ and $b>0$,
(c) or $a>0$ and $b<-1$.

\section{Related inequalities}

Making use of (logarithmically) complete monotonicity of some functions discussed above, we can derive some inequalities for the ratio of two gamma functions and for the divided differences of polygamma functions.

\subsection{First group of inequalities}

From the logarithmically complete monotonicity of the function $\mathcal{H}_{s, t ; \lambda}(x)$ defined in (6.4), the following inequalities were derived in [91, Theorem 1.4].

For $a, b>0$, the inequality

$$
\left[\frac{\Gamma(b)}{\Gamma(a)}\right]^{1 /(b-a)}<\sqrt{a b}\left(\frac{a}{b}\right)^{1 / 2(b-a)}
$$

holds for $0<|b-a|<1$ and reverses for $|b-a|>1$. For $0<|b-a|<1$, the double inequality

$$
\begin{aligned}
& \frac{(k-1) !}{2}\left[\left(\frac{1}{b-a}+\beta\right) \frac{1}{a^{k}}+\left(\beta-\frac{1}{b-a}\right) \frac{1}{b^{k}}\right] \\
& <\frac{(-1)^{k-1}\left[\psi^{(k-1)}(b)-\psi^{(k-1)}(a)\right]}{b-a} \\
& <\frac{(k-1) !}{2}\left[\left(\frac{1}{b-a}+\gamma\right) \frac{1}{a^{k}}+\left(\gamma-\frac{1}{b-a}\right) \frac{1}{b^{k}}\right]
\end{aligned}
$$

holds if and only if $\beta \leq 1$ and $\gamma \geq \frac{1}{|b-a|}$. For $|b-a|>1$, inequalities in (8.1) are valid if and only if $\beta \leq \frac{1}{|b-a|}$ and $\gamma \geq 1$.

\subsection{Second group of inequalities}

From the logarithmically complete monotonicity of the function $H_{s, t ; \lambda}(x)$ defined in (6.5), the following double inequalities were procured in [83, Theorem 1.3] and [108, Theorem 1.5]. 
For $a, b>0$,

1. when $0<|b-a|<1$, the double inequality

$$
\begin{aligned}
\beta_{2} \frac{(k-1) !}{b-a}\left(\frac{1}{a^{k}}-\frac{1}{b^{k}}\right) & <\frac{(-1)^{k-1}\left[\psi^{(k-1)}(b)-\psi^{(k-1)}(a)\right]}{b-a}-\frac{(k-1) !}{2}\left(\frac{1}{a^{k}}+\frac{1}{b^{k}}\right) \\
& <\gamma_{2} \frac{(k-1) !}{b-a}\left(\frac{1}{a^{k}}-\frac{1}{b^{k}}\right)
\end{aligned}
$$

holds if and only if $\beta_{2} \leq \frac{1}{2}$ and $\gamma_{2} \geq 1-\frac{|b-a|}{2}$;

2. when $|b-a|>1$, the double inequality (8.2) is valid if and only if $\beta_{2} \leq 1-\frac{|b-a|}{2}$ and $\gamma_{2} \geq \frac{1}{2}$.

3. when $0<|b-a|<1$, the double inequality

$$
\sqrt{a b}\left(\frac{a}{b}\right)^{\beta_{3} /(b-a)}<\left[\frac{\Gamma(b)}{\Gamma(a)}\right]^{1 /(b-a)}<\sqrt{a b}\left(\frac{a}{b}\right)^{\gamma_{3} /(b-a)}
$$

holds if and only if $\beta_{3} \geq 1-\frac{|b-a|}{2}$ and $\gamma_{3} \leq \frac{1}{2}$;

4. when $|b-a|>1$, the double inequality (8.3) is reversed if and only if $\beta_{3} \leq 1-\frac{|b-a|}{2}$ and $\gamma_{3} \geq \frac{1}{2}$

5. the double inequality

$$
\beta_{1} \frac{k !}{x^{k+1}}<(-1)^{k+1} \psi^{(k)}(x)-\frac{(k-1) !}{x^{k}}<\gamma_{1} \frac{k !}{x^{k+1}}
$$

holds on $(0, \infty)$ if and only if $\beta_{1} \leq \frac{1}{2}$ and $\gamma_{1} \geq 1$.

The inequality (8.3) can be rearranged in the following beautiful form:

$$
\frac{1}{\gamma_{3}} \frac{\ln b-\ln a}{b-a}<\ln \sqrt{a b}-\frac{\ln \Gamma(b)-\ln \Gamma(a)}{b-a}<\frac{1}{\beta_{3}} \frac{\ln b-\ln a}{b-a} .
$$

\section{Some new properties for the ratio of two gamma functions}

In $[64,69,101,106,107,112]$ and the closely related references therein, some kinds of functions involving the ratio of two gamma functions were surveyed. In what follows, we summarize several new kinds of functions involving the ratio of two gamma functions, including some relating to the Catalan numbers in combinatorics and the Bernoulli trials in probability. For information as regards the Catalan numbers, please refer to the survey articles $[93,96]$ and the closely related references therein.

\subsection{An exponential integral representation and logarithmically complete monotonicity}

Let

$$
F_{a, b}(x)=\frac{\Gamma(x+a)}{\Gamma(x+b)} \frac{(x+b)^{x+b-a}}{(x+a)^{x}}, \quad a, b \in \mathbb{R}, a \neq b, x>-\min \{a, b\}
$$

In [54, Theorem 2] and [115, Theorem 3], it was established that, when $a, b>0$, 
1. the function $F_{a, b}(x)$ has the exponential representation

$$
F_{a, b}(x)=\exp \left[b-a+\int_{0}^{\infty} \frac{1}{t}\left(a+\frac{1}{t}-\frac{1}{1-e^{-t}}\right)\left(e^{-b t}-e^{-a t}\right) e^{-x t} \mathrm{~d} t\right]
$$

on $[0, \infty)$;

2. the function $\left[F_{a, b}(x)\right]^{ \pm 1}$ is logarithmically completely monotonic on $[0, \infty)$ if and only if

$$
(a, b) \in D_{ \pm}(a, b)=\{(a, b): a \gtrless b, a \geq 1\} \cup\left\{(a, b): a \lessgtr b, a \leq \frac{1}{2}\right\} .
$$

\subsection{An exponential expansion}

Theorem 3 in [110] reads

$$
\frac{\Gamma(a)}{\Gamma(b)}=\frac{a^{a-1 / 2}}{b^{b-1 / 2}} \exp \left[\sum_{j=1}^{\infty} \frac{B_{2 j}}{2 j(2 j-1)}\left(\frac{1}{a^{2 j-1}}-\frac{1}{b^{2 j-1}}\right)\right], \quad a, b>0 .
$$

\subsection{A double inequality}

Theorem 11 in [110] states that

$$
\begin{aligned}
& \sqrt{\frac{b}{a}}[I(a, b)]^{a-b} \exp \left[\sum_{j=1}^{2 m} \frac{B_{2 j}}{2 j(2 j-1)}\left(\frac{1}{a^{2 j-1}}-\frac{1}{b^{2 j-1}}\right)\right] \\
& <\frac{\Gamma(a)}{\Gamma(b)}<\sqrt{\frac{b}{a}}[I(a, b)]^{a-b} \exp \left[\sum_{j=1}^{2 m-1} \frac{B_{2 j}}{2 j(2 j-1)}\left(\frac{1}{a^{2 j-1}}-\frac{1}{b^{2 j-1}}\right)\right], \quad a, b>0,
\end{aligned}
$$

where $B_{i}$ for $i \in \mathbb{N}$ are the Bernoulli numbers defined [80, 81, 84] by

$$
\frac{x}{e^{x}-1}=\sum_{i=0}^{\infty} B_{i} \frac{x^{i}}{i !}=1-\frac{x}{2}+\sum_{j=1}^{\infty} B_{2 j} \frac{x^{2 j}}{(2 j) !}, \quad|x|<2 \pi
$$

and $I(\alpha, \beta)$ is the exponential mean defined for $\alpha, \beta>0$ by

$$
I(\alpha, \beta)= \begin{cases}\frac{1}{e}\left(\frac{\beta^{\beta}}{\alpha^{\alpha}}\right)^{1 /(\beta-\alpha)}, & \alpha \neq \beta \\ \alpha, & \alpha=\beta .\end{cases}
$$

\subsection{Logarithmically complete monotonicity and applications in probability}

Let $m \in \mathbb{N}, \boldsymbol{a}=\left(a_{1}, a_{2}, \ldots, a_{m}\right)$ with $a_{i}>0$ for $1 \leq i \leq m$, and $\boldsymbol{p}=\left(p_{1}, p_{2}, \ldots, p_{m}\right)$ with $p_{i} \in$ $(0,1)$ for $1 \leq i \leq m$ and $\sum_{i=1}^{m} p_{i}=1$. In [57,113], it was proved that the function

$$
\mathcal{Q}_{\boldsymbol{a}, \boldsymbol{p} ; m}(x)=\frac{\Gamma\left(1+x \sum_{i=1}^{m} a_{i}\right)}{\prod_{i=1}^{m} \Gamma\left(1+x a_{i}\right)} \prod_{i=1}^{m} p_{i}^{x a_{i}}
$$

is logarithmically completely monotonic on $(0, \infty)$. This is a generalization of a problem originating from the Bernoulli trials in probability (see [6, 52]). This logarithmically complete monotonicity can be used to derive some inequalities for multinomial coefficients and multivariate beta functions (see $[6,113]$ ). 
In [79], the $q$-analog of the function (9.1) was considered and its logarithmically complete monotonicity was investigated.

At the deep night on 6 May 2018, Dr. Frédéric Ouimet (Université de Montréal, Canada) acknowledged that, in his arXiv preprint [57], which was formally published as [58] later, he alternatively and essentially proved [113, Theorem 2.2] and applied it to deriving asymptotic formulas for quantities of interest in the context of a statistical density estimation based on the Bernstein polynomials on the $n$-dimensional simplex.

\subsection{Completely monotonic degrees of functions involving ratios of gamma functions}

Recall from [125] that a function $f$ is said to be strongly completely monotonic on $(0, \infty)$ if it has derivatives of all orders and $(-1)^{n} x^{n+1} f^{(n)}(x)$ is nonnegative and decreasing on $(0, \infty)$ for all $n \geq 0$.

Let

$$
L_{s, t}(x)=x-\frac{\Gamma(x+t)}{\Gamma(x+s)} x^{s-t+1}=x\left[1-\frac{\Gamma(x+t) / x^{t}}{\Gamma(x+s) / x^{s}}\right]
$$

and suppose that either

1. $s \geq 1>t$ and $0<s-t \leq \frac{1}{2}$, or

2. $t \geq 1$ and $0<s-t<1$, or

3. $\frac{\sqrt{3}}{6}+\frac{1}{2} \leq t<s<1$.

Theorem 1 in [47] reads

1. the function

$$
\Phi_{s, t}(x)=\frac{L_{s, t}^{\prime \prime}(x)}{L_{s, t}(x)-x}=-\frac{\Gamma(x+s)}{\Gamma(x+t)} x^{t-s-1} L_{s, t}^{\prime \prime}(x)
$$

is strongly completely monotonic on $(0, \infty)$;

2. the function $L_{s, t}(x)$ is strictly increasing and concave on $(0, \infty)$ and the function

$-L^{\prime \prime}(x)$ is completely monotonic on $(0, \infty)$.

Proposition 1.1 in [50, p. 34], which was proved in [65, Sect. 4.2], states that a function $f(x)$ is strongly completely monotonic if and only if the function $x f(x)$ is completely monotonic. In other words, the set of functions of completely monotonic degree not less than 1 with respect to $x \in(0, \infty)$ coincides with the set of strongly completely monotonic functions on $(0, \infty)$. This implies that $\operatorname{deg}_{\mathrm{cm}}^{x}[\Phi(x)] \geq 1$.

Corollary 3 in [47] can be restated as follows: the functions

$$
\Delta(x), \quad \frac{2}{3}\left[\psi^{\prime}(x)+\frac{1}{2 x}\right]^{2}+\psi^{\prime \prime}(x)-\frac{1}{2 x^{2}}, \quad \text { and } \quad-\psi^{\prime \prime}(x)-\frac{2}{x} \psi^{\prime}(x)+\frac{1}{x^{2}}
$$

are of completely monotonic degree not less than 2. In [48, Theorem 1.3], the first two functions above were proved to be of completely monotonic degree at least 3 , but the third function is of completely monotonic degree less than 3 .

In $[48,49]$, the functions $L_{s, t}(x)$ and $\Phi_{s, t}(x)$ were further investigated and more conclusions were discovered therein.

From the identity (3.16), we surely conclude that main results and their proofs in [47-49] can be improved. We also believe that there should be a simple method or a nice approach 
to find some properties of the functions $L_{s, t}(x)$ and $\Phi_{s, t}(x)$. For this purpose, the integral representation

$$
\frac{\Gamma(x+a)}{\Gamma(x+b)}=\frac{1}{\Gamma(b-a)} \int_{0}^{\infty}\left(1-e^{-t}\right)^{b-a-1} e^{-(x+a) t} \mathrm{~d} t
$$

for $\Re(b-a)>0$ and $\Re(x+a)>0$, which can be found in [10, p. 165] and [124, p. 67], would play a key role.

\subsection{Necessary and sufficient conditions}

In [129], Theorems 3.1 and 3.2 show that

1. the function

$$
v(s, t, r ; x)= \begin{cases}e^{-\psi(x+r)}\left[\frac{\Gamma(x+t)}{\Gamma(x+s)}\right]^{1 /(t-s)}, & t \neq s, \\ e^{\psi(x+s)-\psi(x+r)}, & t=s\end{cases}
$$

is logarithmically completely monotonic with respect to $x \in(-\rho, \infty)$ if and only if $r \leq \min \{s, t\}$ and its reciprocal $\frac{1}{v(s, t, r ; x)}$ is logarithmically completely monotonic with respect to $x \in(-\rho, \infty)$ if and only if $r \geq \frac{s+t}{2}$, where $s, t, r \in \mathbb{R}$ and $\rho=\min \{s, t, r\}$; see also [131, Corollary 3];

2. the function

$$
U(a, b, c ; x)= \begin{cases}\frac{1}{x+c}\left[\frac{\Gamma(x+a)}{\Gamma(x+b)}\right]^{1 /(a-b)}, & a \neq b, \\ \frac{1}{x+c} e^{\psi(x+a)}, & a=b,\end{cases}
$$

is logarithmically completely monotonic with respect to $x \in(-\rho, \infty)$ if and only if $c \leq \frac{a+b-\max \{|a-b|, 1\}}{2}$ and its reciprocal $\frac{1}{U(a, b, c ; x)}$ is logarithmically completely monotonic with respect to $x \in(-\rho, \infty)$ if and only if $c \geq \frac{a+b-\min \{|a-b|, 1\}}{2}$, where $a, b, c \in \mathbb{R}$ and $\rho=\min \{a, b, c\}$.

\subsection{Five classes of functions involving ratios of gamma functions}

In [133], the logarithmically complete monotonicity of the functions

$$
\begin{aligned}
& \frac{W_{r, s}(x)}{W_{u, v}(x)}, \quad \frac{W_{\bar{r}, s}(x)}{\prod_{i=1}^{n} W_{r_{i}, s_{i}}^{\lambda_{i}}(x)}, \quad \frac{\prod_{i=1}^{n} \Gamma^{\lambda_{i}}\left(x+s_{i}\right)}{\Gamma(x+s)}, \\
& \prod_{i=1}^{n} \frac{\Gamma\left(x+u_{i}\right)}{\Gamma\left(x+r_{i}\right)}, \quad \prod_{i=1}^{n} \frac{W_{r_{i}, s}(x)}{W_{u_{i}, s}(x)}
\end{aligned}
$$

was investigated, where $W_{u, v}(x)$ is defined in (2.1). Concretely speaking,

1. Theorem 3.1 in [133] states that, for $u, v, r, s \in \mathbb{R}$ and $\rho=\min \{u, v, r, s\}$, the function $\frac{W_{r, s}(x)}{W_{u, v}(x)}$ is logarithmically completely monotonic on $(-\rho, \infty)$ if and only if $u+v \leq r+s$ and $\min \{u, v\} \leq \min \{r, s\}$; see also [131, Proposition 1] and [132, Corollary 3];

2. Theorem 3.7 in [133] states that, if $r, s, r_{i}, s_{i} \in \mathbb{R}$ and $\lambda_{i} \geq 0$ such that $\sum_{i=1}^{n} \lambda_{i}=1$, then (a) the function $\frac{W_{\bar{r}, \bar{s}}(x)}{\prod_{i=1}^{n} W_{r_{i}, s_{i}}^{\lambda_{i}(x)}}$ is logarithmically completely monotonic on $\left(-\rho_{1}, \infty\right)$, where $\rho_{1}=\min \left\{r_{i}, s_{i}: 1 \leq i \leq n\right\}$ and $(\bar{r}, \bar{s})=\left(\sum_{i=1}^{n} \lambda_{i} r_{i}, \sum_{i=1}^{n} \lambda_{i} s_{i}\right)$; 
(b) the function $\frac{W_{r, s}(x)}{\prod_{i=1}^{n} W_{r, s_{i}}^{\lambda}(x)}$ is logarithmically completely monotonic on $\left(-\rho_{2}, \infty\right)$ if and only if $s \geq \bar{s}$;

(c) the function $\frac{\prod_{i=1}^{n} W_{r, s}^{\lambda_{i}}(x)}{W_{r, s}(x)}$ is logarithmically completely monotonic on $\left(-\rho_{2}, \infty\right)$ if and only if $s \leq \min \left\{s_{i}: 1 \leq i \leq n\right\}$, where $\rho_{2}=\min \left\{r, s, s_{i}: 1 \leq i \leq n\right\}$;

3. Theorems 4.2 and 4.3 in [133] show that, if $\lambda_{i}>0$ such that $\sum_{i=0}^{n} \lambda_{i}=1$, then

(a) the function $\frac{\prod_{i=1}^{n} \Gamma^{\lambda_{i}\left(x+s_{i}\right)}}{\Gamma(x+s)}$ is greater than 1 and logarithmically completely monotonic on $\left(-\min \left\{s, s_{i}: 1 \leq i \leq n\right\}, \infty\right)$ if and only if $s=\bar{s}$;

(b) the function $\frac{\Gamma(x+s)}{\prod_{i=1}^{n} \Gamma^{\lambda} i\left(x+s_{i}\right)}$ is logarithmically completely monotonic on $\left(-\min \left\{s, s_{i}: 1 \leq i \leq n\right\}, \infty\right)$ if and only if $s \leq \min \left\{s_{i}: 1 \leq i \leq n\right\}$;

4. Theorems 4.7, 4.9, and 4.12 in [133] show that, if $u_{1} \leq u_{2} \leq \cdots \leq u_{n}$ and $r_{1} \leq r_{2} \leq \cdots \leq r_{n}$ such that $\sum_{i=1}^{k} u_{i} \leq \sum_{i=1}^{k} r_{i}$ for $k=2, \ldots, n-1$, then

(a) the function $\prod_{i=1}^{n} \frac{\Gamma\left(x+u_{i}\right)}{\Gamma\left(x+r_{i}\right)}$ is logarithmically completely monotonic on $\left(-\min \left\{u_{1}, r_{1}\right\}, \infty\right)$ if and only if $u_{1} \leq r_{1}$ and $\sum_{i=1}^{n} u_{i} \leq \sum_{i=1}^{n} r_{i}$

(b) the function $\ln \prod_{i=1}^{n} \frac{\Gamma\left(x+u_{i}\right)}{\Gamma\left(x+r_{i}\right)}$ is completely monotonic on the interval $\left(-\min \left\{u_{1}, r_{1}\right\}, \infty\right)$ if and only if $u_{1} \leq r_{1}$ and $\sum_{i=1}^{n} u_{i}=\sum_{i=1}^{n} r_{i}$;

(c) the function $\ln \prod_{i=1}^{n} \frac{W_{r_{i}, s}(x)}{W_{u_{i}, s}(x)}$ is completely monotonic on the interval $\left(-\min \left\{u_{1}, r_{1}, s\right\}, \infty\right)$ if and only if $\sum_{i=1}^{n} u_{i} \leq \sum_{i=1}^{n} r_{i}$.

\subsection{Complete monotonicity of a multivariate function involving ratios of the gamma functions}

It is well known [16, Theorem 4.2.2] that a function $f\left(x_{1}, x_{2}, \ldots, x_{k}\right)$ is called completely monotonic in a cone $X=X_{1} \times X_{2} \times \cdots \times X_{k}$ if it is $C^{\infty}$ and

$$
(-1)^{n_{1}+n_{2}+\cdots+n_{k}} \frac{\partial^{n_{1}+n_{2}+\cdots+n_{k}} f\left(x_{1}, x_{2}, \ldots, x_{k}\right)}{\partial x_{1}^{n_{1}} \partial x_{2}^{n_{2}} \cdots \partial x_{k}^{n_{k}}} \geq 0
$$

for all $n_{1}, n_{2}, \ldots, n_{k} \geq 0$. See also [55, Chapter XIII, Sect. 6] and the closely related references therein. For $x, y>-\min \{p, q\}$, let

$$
G(p, q ; x, y)= \begin{cases}{\left[\frac{\Gamma(x+p) \Gamma(y+q)}{\Gamma(y+p) \Gamma(x+q)}\right] / /(p-q)(x-y),} & (p-q)(x-y) \neq 0 ; \\ \exp \left[\frac{\psi(x+p)-\psi(y+p)}{x-y}\right], & p=q, x \neq y ; \\ \exp \left[\frac{\psi(x+p)-\psi(x+q)}{p-q}\right], & p \neq q, x=y ; \\ e^{\psi^{\prime}(x+p),} & p=q, x=y .\end{cases}
$$

Then

1. Theorem 1 in [132] states that the function $\ln G(p, q ; x, y)$ is completely monotonic on $\Omega=\{(p, q, x, y): x, y>-\min \{p, q\}, p, q, x, y \in \mathbb{R}\} ;$

2. Theorem 2 in [132] states that, for fixed $p, q, r, s \in \mathbb{R}$ and $\rho=\min \{p, q, r, s\}$, the function $(x, y) \mapsto \ln \frac{G(p, q ; x, y)}{G(r, s ; x, y)}$ is completely monotonic on $(-\rho, \infty)^{2}$ if and only if $p+q \leq r+s$ and $\min \{p, q\} \leq \min \{r, s\}$.

\section{Difference between trigamma and exponential functions}

In [33, Lemma 2], the inequality

$$
\psi^{\prime}(x)<e^{1 / x}-1, \quad x>0,
$$

was obtained and applied. Hereafter, there have been some developments on this topic. 
In [82, Theorem 3.1], [116, Theorem 1.1], and [118], among other things, by different and elementary approaches, the inequality (10.1) was generalized to complete monotonicity, which shows that the difference

$$
h(x)=e^{1 / x}-\psi^{\prime}(x)
$$

is completely monotonic on $(0, \infty)$.

For $\alpha, \beta>0$, let

$$
h_{\alpha, \beta}(x)=\alpha e^{\beta / t}-\psi^{\prime}(x)
$$

on $(0, \infty)$. Theorem 4.1 in [82] reads

1. the function $h_{1, \beta}(x)$ is completely monotonic on $(0, \infty)$ if and only if $\beta \geq 1$;

2. if $\beta \geq 1$ and $\alpha \beta \geq 1$, the function $h_{\alpha, \beta}(x)$ is completely monotonic on $(0, \infty)$;

3. a necessary condition for the function $h_{\alpha, \beta}(x)$ to be completely monotonic on $(0, \infty)$ is $\alpha \beta \geq 1$;

4. if $0<\beta<1$, the condition

$$
\alpha \beta \geq \max _{u \in(0, \infty)} F_{\beta}(u)>1
$$

is necessary and sufficient for $h_{\alpha, \beta}(x)$ to be completely monotonic on $(0, \infty)$, where

$$
\lim _{u \rightarrow 0^{+}} F_{\beta}(u)=1 \quad \text { and } \quad \lim _{u \rightarrow \infty} F_{\beta}(u)=0
$$

for all $\beta>0$.

For $\alpha, \beta>0$, let

$$
H_{\alpha, \beta}(x)=h_{\alpha, \beta}(x)-\alpha
$$

on $(0, \infty)$. Theorem 1.3 in [75] states that

1. if $(\alpha, \beta)=(1,1)$, then

$$
\operatorname{deg}_{\mathrm{cm}}^{x}\left[H_{1,1}(x)\right]=4
$$

2. if $\beta>1$, then

$$
\operatorname{deg}_{\mathrm{cm}}^{x}\left[H_{1 / \beta, \beta}(x)\right]=2 ;
$$

3. if $\alpha \beta>1$ and $\beta \geq 1$, or if $\alpha \beta>1,0<\beta<1$, and

$$
\alpha \beta^{2} \geq \max _{u \in(0, \infty)}\left\{G_{\beta}(u)\right\}
$$

then

$$
\operatorname{deg}_{\mathrm{cm}}^{x}\left[H_{\alpha, \beta}(x)\right]=1
$$


In [130, Theorem 1] that the double inequality

$$
\frac{1}{x^{2}}+\theta(x, p)<\psi^{\prime}(x)<\frac{1}{x^{2}}+\theta(x, q), \quad x>0,
$$

was proved to be valid if and only if $p \leq 1$ and $q \geq 2$, where

$$
\theta(x, m)=\frac{e^{m /(x+1)}-e^{-m / x}}{2 m}
$$

This means that

$$
\sinh \frac{1}{x+1}<\psi^{\prime}(x+1)<\frac{1}{2} \sinh \frac{2}{x}, \quad x>0 .
$$

In [111], when $x>3$, the inequality (10.1) was refined as

$$
e^{m(x)}-1<\psi^{\prime}(x)<e^{M(x)}-1,
$$

where

$$
m(x)=\frac{1}{x}-\frac{1}{24 x^{4}}+\frac{7}{360 x^{6}} \quad \text { and } \quad M(x)=m(x)+\frac{1}{90 x^{7}} .
$$

Are the functions

$$
\psi^{\prime}(x+1)-\sinh \frac{1}{x+1} \quad \text { and } \quad \frac{1}{2} \sinh \frac{2}{x}-\psi^{\prime}(x+1)
$$

completely monotonic on $(0, \infty)$ ?

By the way, we note the function $e^{ \pm 1 / x}$ has something to do with the existence of partitions of unity in differential geometry and with the Lah numbers in combinatorics. For more information, please refer to $[17,22,35,37,44,70,74,77,78,95,104,121,134]$ and the closely related references therein.

\section{Remarks}

\subsection{First remark}

In $[45$, p. 20], Kazarinoff proved the inequality

$$
[\ln \phi(t)]^{\prime \prime}-\left\{[\ln \phi(t)]^{\prime}\right\}^{2}>0
$$

where

$$
\phi(t)=\int_{0}^{\pi / 2} \sin ^{t} x \mathrm{~d} x=\frac{\sqrt{\pi}}{2} \frac{\Gamma((t+1) / 2)}{\Gamma((t+2) / 2)}
$$

for $-1<t<\infty$. We observe that the inequality (11.1) can be rewritten as

$$
\left[\frac{\psi((t+2) / 2)-\psi((t+1) / 2)}{(t+2) / 2-(t+1) / 2}\right]^{2}+2 \frac{\psi^{\prime}((t+2) / 2)-\psi^{\prime}((t+1) / 2)}{(t+2) / 2-(t+1) / 2}<0
$$


for $t>-1$. Letting $u=\frac{t+1}{2}$ in (11.2) yields

$$
\left[\frac{\psi(u+1 / 2)-\psi(u)}{(u+1 / 2)-u}\right]^{2}+2 \frac{\psi^{\prime}(u+1 / 2)-\psi^{\prime}(u)}{(u+1 / 2)-u}<0
$$

for $u>0$. Consequently, it is easy to see that the inequality (11.2) or (11.3) can be derived from complete monotonicity of the function $\Delta_{s, t ; \lambda}(x)$ defined in (4.1).

\subsection{Second remark}

In the proof of [19, Theorem 1.1], by using the convolution theorem for Laplace transforms, the inequality

$$
\psi^{\prime}(x+b)-\psi^{\prime}(x+a)+\frac{1}{b-a}[\psi(x+b)-\psi(x+a)]^{2}>0,
$$

which is equivalent to

$$
\left[\frac{\psi(x+b)-\psi(x+a)}{b-a}\right]^{2}+\frac{\psi^{\prime}(x+b)-\psi^{\prime}(x+a)}{b-a}>0,
$$

for $0 \leq a<b<a+1$ was proved. This result can be directly deduced from complete monotonicity of the functions $\Delta_{s, t}(x)$ and $\Delta_{s, t ; \lambda}(x)$.

\subsection{Third remark}

In [5, Lemma 7], the inequality

$$
\psi^{\prime}(x)-\psi^{\prime}(x+c)>[\psi(x+c)-\psi(x)]^{2}
$$

which can be rearranged as

$$
\left[\frac{\psi(x+c)-\psi(x)}{(x+c)-x}\right]^{2}-\frac{1}{c} \frac{\psi^{\prime}(x+c)-\psi^{\prime}(x)}{(x+c)-x}<0
$$

on $(0, \infty)$ for $0<c<1$ and its reversed version for $c>1$ were proved. This inequality is also a special case of complete monotonicity of the function $\Delta_{s, t ; \lambda}(x)$ defined in (4.1).

\subsection{Fourth remark}

In [24, p. 13], the following inequalities were obtained:

$$
\begin{aligned}
& \frac{1}{c}[\psi(x+c)-\psi(x)]^{2}>\psi^{\prime}(x)-\psi^{\prime}(x+c)>[\psi(x+c)-\psi(x)]^{2} \\
& \frac{1-q}{1-q^{c}}\left[\psi_{q}(x+c)-\psi_{q}(x)\right]^{2}>q^{x}\left[\psi_{q}^{\prime}(x)-\psi_{q}^{\prime}(x+c)\right] \\
&>\left[\psi_{q}(x+c)-\psi_{q}(x)\right]^{2}
\end{aligned}
$$

where $0<c<1,0<q<1$, and $x>0$. When $c>1$, the inequalities in (11.4) and (11.5) reverse. It is clear that both inequalities in (11.4) are special cases of complete monotonicity of the function $\Delta_{s, t ; \lambda}(x)$. 
The inequalities (11.4) and (11.5) can be reformulated as

$$
-\left[\frac{\psi(x+c)-\psi(x)}{(x+c)-x}\right]^{2}<\frac{\psi^{\prime}(x+c)-\psi^{\prime}(x)}{(x+c)-x}<-c\left[\frac{\psi(x+c)-\psi(x)}{(x+c)-x}\right]^{2}
$$

and

$$
\begin{aligned}
-\frac{c}{q^{x}} \frac{1-q}{1-q^{c}}\left[\frac{\psi_{q}(x+c)-\psi_{q}(x)}{(x+c)-x}\right]^{2} & <\frac{\psi_{q}^{\prime}(x+c)-\psi_{q}^{\prime}(x)}{(x+c)-x} \\
& <-\frac{c}{q^{x}}\left[\frac{\psi_{q}(x+c)-\psi_{q}(x)}{(x+c)-x}\right]^{2} .
\end{aligned}
$$

Stimulated by (11.5), we naturally ask a question: what are the $q$-analogs of the function $\Delta_{s, t ; \lambda}(x)$ ? Are they completely monotonic functions?

\subsection{Fifth remark}

For $\alpha, \beta \in \mathbb{R}$ satisfying $\alpha \neq \beta$ and $(\alpha, \beta) \notin\{(0,1),(1,0)\}$, let

$$
q_{\alpha, \beta}(t)= \begin{cases}\frac{e^{\beta t}-e^{\alpha t}}{e^{t}-1}, & t \neq 0 \\ \beta-\alpha, & t=0\end{cases}
$$

It is clear that $q_{\alpha, 0}(t)=-\alpha h_{\alpha}(-t)$, or say, $h_{\alpha}(t)=-\frac{q_{\alpha, 0}(-t)}{\alpha}$, where $h_{\alpha}(t)$ is defined by (3.9). This implies that some properties of the function $q_{\alpha, \beta}(t)$ can be applied in [128].

The function $q_{\alpha, \beta}(t)$ plays an important role in some investigations on functions involving the ratio of two gamma functions. For more details, please refer to [64, Sect. 1.7], [106, Sect. 4.1], the survey articles [107, 109], [26, 31, 47, 48, 60, 61, 87, 99], and the closely related references therein.

\subsection{Sixth remark}

For more information on the history and properties of logarithmically completely monotonic functions, please refer to [15], [27, pp. 21-23], [64, pp. 5-6, Sect. 1.5], [100, pp. 21542155 , Remark 8], and the closely related references therein. The notion "logarithmically completely monotonic function" has been collected in the monograph [122]: see [122, Definition 5.10], [122, Theorem 5.11], [122, Proposition 5.17], [122, Proposition 5.25], [122, Comments 5.29], and [122, Remark 9.18].

\section{Open problems}

Finally, we would like to pose more open problems.

\subsection{First problem}

What are the $q$-analogs of $\Delta_{s, t}(x), \Delta_{s, t ; \lambda}(x), \delta_{s, t}(x), \delta_{s, t ; \lambda}(x), \theta_{s, t ; \lambda}(x)$, and others in this paper? What about the (logarithmically) complete monotonicity of these $q$-analogs?

\subsection{Second problem}

Can one find integral representations of the form in (1.1) for the (logarithmically) completely monotonic functions collected in this paper? 


\subsection{Third problem}

Motivated by [89, p. 75, Corollary 2] and others, we can naturally generalize the function (2.1) to

$$
f_{p, q ; s, t}(x)=\left[\frac{\Gamma(x+t)}{\Gamma(x+s)}\right]^{p /(t-s)}-q x
$$

on $(-\alpha, \infty)$, where $0<|t-s| \neq 1, \alpha=\min \{s, t\}, p \neq 0$, and $q \in \mathbb{R}$. How about the monotonicity and convexity of the function $f_{p, q ; s, t}(x)$ ?

\subsection{Fourth problem}

Motivated by the results in [40], we guess that the difference between the right and left hand sides of $(2.5)$ is a completely monotonic function on $(0, \infty)$.

\subsection{Fifth problem}

For $m, n \in \mathbb{N}$ and $i, j \in \mathbb{N}$, let

$$
f_{m, n ; i, j}(x)=\left[\psi^{(m)}(x)\right]^{i}+\left[\psi^{(n)}(x)\right]^{j}
$$

on $(0, \infty)$. It is clear that $f_{1,2 ; 2,1}(x)=f_{1,2}(x)$, a special case of the function (3.15), and that $f_{m, n ; i, j}(x)=f_{n, m ; j, i}(x)$. Stimulated by [38], we pose to discuss complete monotonicity of the function (12.1).

\subsection{Sixth problem}

For $n \in \mathbb{N}$, let

$$
f_{n}(x)=\sum_{k m=n}\left[\psi^{(k)}(x)\right]^{m}
$$

on $(0, \infty)$. It is clear that

$$
\begin{aligned}
& f_{1}(x)=\psi^{\prime}(x), \quad f_{2}(x)=\Delta(x), \quad f_{3}(x)=\left[\psi^{\prime}(x)\right]^{3}+\psi^{\prime \prime \prime}(x), \\
& f_{4}(x)=\left[\psi^{\prime}(x)\right]^{4}+\left[\psi^{\prime \prime}(x)\right]^{2}+\psi^{(4)}(x), \quad f_{5}(x)=\left[\psi^{\prime}(x)\right]^{5}+\psi^{(5)}(x), \\
& f_{6}(x)=\left[\psi^{\prime}(x)\right]^{6}+\left[\psi^{\prime \prime}(x)\right]^{3}+\left[\psi^{(3)}(x)\right]^{2}+\psi^{(6)}(x) .
\end{aligned}
$$

For $\ell \in \mathbb{N}$, prove that the functions $f_{2 \ell-1}(x)$ is completely monotonic on $(0, \infty)$, but $f_{2 \ell}(x)$ is not. This problem was suggested by Dr. Li Yin (Binzhou University, Shangdong, China) on 26 August 2018.

\subsection{Seventh problem}

We conjectured [72, Remark 6] that the double inequality

$$
\frac{1}{x^{4}}\left[\frac{x^{2}+4 x+12}{12(x+1)^{2}}\right]^{\alpha}<\left[\psi^{\prime}(x)\right]^{2}+\psi^{\prime \prime}(x)<\frac{1}{x^{4}}\left[\frac{x^{2}+4 x+12}{12(x+1)^{2}}\right]^{\beta}
$$

holds on $(0, \infty)$ if and only if $\alpha \geq \frac{6}{5}$ and $\beta \leq 1$. 


\subsection{Eighth problem}

\section{The function $\frac{1}{\arctan x}$ is logarithmically completely monotonic on $(0, \infty)$, but not a Stieltjes} transform. This problem was first proposed in 2010.

\section{Acknowledgements}

This is a revised, updated, and extended version of the talk reported between 10:30-11:30, Thursday, September 2, 2010 in the Workshop on Integral Transforms, Positivity and Applications, held at University of Copenhagen. The first author appreciates three organizers (Professor Christian Berg, Assistant Professor Jacob Stordal Christiansen, and Associate Professor Henrik Laurberg Pedersen) of the workshop for their inviting to be as a plenary speaker of the workshop, for their full covering of expanses, and for their hospitality during the whole trip to attend the workshop. The workshop is sponsored by the Danish Research Council and Nordforsk. The authors appreciate the anonymous referees for their careful corrections to and valuable comments on the original version of this paper.

\section{Funding}

Not applicable.

\section{Competing interests}

The authors declare that they have no competing interests.

\section{Authors' contributions}

All authors contributed equally to the manuscript and read and approved the final manuscript.

\section{Author details}

${ }^{1}$ Institute of Mathematics, Henan Polytechnic University, Jiaozuo, China. ${ }^{2}$ College of Mathematics, Inner Mongolia University for Nationalities, Tongliao, China. ${ }^{3}$ School of Mathematical Sciences, Tianjin Polytechnic University, Tianjin, China. ${ }^{4}$ Department of Mathematics, Texas A\&M University-Kingsville, Kingsville, USA. ${ }^{5}$ Florida Institute of Technology, Melbourne, USA.

\section{Publisher's Note}

Springer Nature remains neutral with regard to jurisdictional claims in published maps and institutional affiliations.

Received: 21 August 2018 Accepted: 16 January 2019 Published online: 06 February 2019

\section{References}

1. Abramowitz, M., Stegun, I.A. (eds.): Handbook of Mathematical Functions with Formulas, Graphs, and Mathematical Tables, 10th printing edn. National Bureau of Standards, Applied Mathematics Series, vol. 55. Dover, New York (1972)

2. Alzer, H.: On some inequalities for the gamma and psi functions. Math. Comput. 66(217), 373-389 (1997). Available online at https://doi.org/10.1090/S0025-5718-97-00807-7

3. Alzer, H.: Sharp inequalities for the digamma and polygamma functions. Forum Math. 16(2), 181-221 (2004) Available online at https://doi.org/10.1515/form.2004.009

4. Alzer, H.: Sharp inequalities for the harmonic numbers. Expo. Math. 24(4), 385-388 (2006). Available online at https://doi.org/10.1016/j.exmath.2006.02.001

5. Alzer, H.: Sub- and superadditive properties of Euler's gamma function. Proc. Am. Math. Soc. 135(11), 3641-3648 (2007). Available online at https://doi.org/10.1090/S0002-9939-07-09057-0

6. Alzer, H.: Complete monotonicity of a function related to the binomial probability. J. Math. Anal. Appl. 459(1), 10-15 (2018). Available online at https://doi.org/10.1016/j.jmaa.2017.10.077

7. Alzer, H., Grinshpan, A.Z.: Inequalities for the gamma and q-gamma functions. J. Approx. Theory 144(1), 67-83 (2007). Available online at https://doi.org/10.1016/j.jat.2006.04.008

8. Alzer, H., Wells, J.: Inequalities for the polygamma functions. SIAM J. Math. Anal. 29(6), 1459-1466 (1998). Available online at https://doi.org/10.1137/S0036141097325071

9. Andrews, G.E.: $q$-Series: Their Development and Application in Analysis, Number Theory, Combinatorics, Physics and Computer Algebra. CBMS Regional Conference Series in Mathematics, vol. 66. Am. Math. Soc., Providence (1986) Published for the Conference Board of the Mathematical Sciences, Washington, DC

10. Andrews, G.E., Askey, R.A., Roy, R.Special Functions. Encyclopedia of Mathematics and Its Applications, vol. 71 Cambridge University Press, Cambridge (1999). Available online at https://doi.org/10.1017/CBO9781107325937

11. Atanassov, R.D., Tsoukrovski, U.V.: Some properties of a class of logarithmically completely monotonic functions. C. R. Acad. Bulgare Sci. 41(2), $21-23$ (1988)

12. Batır, N.: An interesting double inequality for Euler's gamma function. J. Inequal. Pure Appl. Math. 5(4), Article ID 97 (2004). Available online at http://www.emis.de/journals/JPAM/article452.html

13. Batı, N.: Some new inequalities for gamma and polygamma functions. J. Inequal. Pure Appl. Math. 6(4), Article ID 103 (2005). Available online at http://www.emis.de/journals/JPAM/article577.htm/

14. Batır, N.: On some properties of digamma and polygamma functions. J. Math. Anal. Appl. 328(1), 452-465 (2007) Available online at https://doi.org/10.1016/j.jmaa.2006.05.065

15. Berg, C.: Integral representation of some functions related to the gamma function. Mediterr. J. Math. 1(4), 433-439 (2004). Available online at https://doi.org/10.1007/s00009-004-0022-6

16. Bochner, S.: Harmonic Analysis and the Theory of Probability. California Monographs in Mathematical Sciences. University of California Press, Berkeley (1955)

17. Boyadzhiev, K.N.: Lah numbers, Laguerre polynomials of order negative one, and the $n$th derivative of $\exp (1 / x)$. Acta Univ. Sapientiae Math. 8(1), 22-31 (2016). Available online at https://doi.org/10.1515/ausm-2016-0002 
18. Bullen, P.S.: Handbook of Means and Their Inequalities. Mathematics and Its Applications, vol. 560. Kluwer Academic, Dordrecht (2003)

19. Chen, C.-P.: Monotonicity and convexity for the gamma function. J. Inequal. Pure Appl. Math. 6(4), Article ID 100 (2005). Available online at http://www.emis.de/journals/JPAM/article574.html

20. Chen, C.P., Qi, F.: Logarithmically completely monotonic functions relating to the gamma function. J. Math. Anal. Appl. 321(1), 405-411 (2006). Available online at https://doi.org/10.1016/j.jmaa.2005.08.056

21. Chen, C.P., Qi, F., Srivastava, H.M.: Some properties of functions related to the gamma and psi functions. Integral Transforms Spec. Funct. 21(2), 153-164 (2010). Available online at https://doi.org/10.1080/10652460903064216

22. Daboul, S., Mangaldan, J., Spivey, M.Z., Taylor, P.J.: The Lah numbers and the $n$th derivative of $e^{1 / x}$. Math. Mag. 86(1), 39-47 (2013). Available online at https://doi.org/10.4169/math.mag.86.1.039

23. Elezović, N., Giordano, C., Pečarić, J.: The best bounds in Gautschi's inequality. Math. Inequal. Appl. 3(2), 239-252 (2000). Available online at https://doi.org/10.7153/mia-03-26

24. Gao, P.: Some monotonicity properties of gamma and q-gamma functions. ISRN Math. Anal. 2011, Article ID 375715 (2011)

25. Gasper, G., Rahman, M.: Basic Hypergeometric Series, 2nd edn. Encyclopedia of Mathematics and Its Applications, vol. 96. Cambridge University Press, Cambridge (2004). Available online at https://doi.org/10.1017/CBO9780511526251

26. Guo, B.-N., Qi, F.: Properties and applications of a function involving exponential functions. Commun. Pure Appl. Anal. 8(4), 1231-1249 (2009). Available online at https://doi.org/10.3934/cpaa.2009.8.1231

27. Guo, B.-N., Qi, F.: A property of logarithmically absolutely monotonic functions and the logarithmically complete monotonicity of a power-exponential function. Sci. Bull. "Politeh." Univ. Buchar., Ser. A, Appl. Math. Phys. 72(2), 21-30 (2010)

28. Guo, B.-N., Qi, F.: Some properties of the psi and polygamma functions. Hacet. J. Math. Stat. 39(2), 219-231 (2010)

29. Guo, B.-N., Qi, F.: Two new proofs of the complete monotonicity of a function involving the psi function. Bull. Korean Math. Soc. 47(1), 103-111 (2010). Available online at https://doi.org/10.4134/bkms.2010.47.1.103

30. Guo, B.-N., Qi, F.: A class of completely monotonic functions involving divided differences of the psi and tri-gamma functions and some applications. J. Korean Math. Soc. 48(3), 655-667 (2011). Available online at https://doi.org/10.4134/JKMS.2011.48.3.655

31. Guo, B.-N., Qi, F.: An alternative proof of Elezović-Giordano-Pečarić's theorem. Math. Inequal. Appl. 14(1), 73-78 (2011). Available online at https://doi.org/10.7153/mia-14-06

32. Guo, B.-N., Qi, F.: A completely monotonic function involving the tri-gamma function and with degree one. Appl. Math. Comput. 218(19), 9890-9897 (2012). Available online at https://doi.org/10.1016/j.amc.2012.03.075

33. Guo, B.-N., Qi, F.: Refinements of lower bounds for polygamma functions. Proc. Am. Math. Soc. 141(3), 1007-1015 (2013). Available online at https://doi.org/10.1090/S0002-9939-2012-11387-5

34. Guo, B.-N., Qi, F.: Sharp inequalities for the psi function and harmonic numbers. Analysis (Berlin) 34(2), 201-208 (2014). Available online at https://doi.org/10.1515/anly-2014-0001

35. Guo, B.-N., Qi, F.: Some integral representations and properties of Lah numbers. J. Algebra Number Theory Acad. 4(3), 77-87 (2014)

36. Guo, B.-N., Qi, F.: On the degree of the weighted geometric mean as a complete Bernstein function. Afr. Math. 26(7), 1253-1262 (2015). Available online at https://doi.org/10.1007/s13370-014-0279-2

37. Guo, B.-N., Qi, F.: Six proofs for an identity of the Lah numbers. Online J. Anal. Comb. 10, 5 pages (2015)

38. Guo, B.-N., Qi, F., Srivastava, H.M.: Some uniqueness results for the non-trivially complete monotonicity of a class of functions involving the polygamma and related functions. Integral Transforms Spec. Funct. 21(11), 849-858 (2010) Available online at https://doi.org/10.1080/10652461003748112

39. Guo, B.-N., Qi, F., Zhao, J.-L., Luo, Q.-M.: Sharp inequalities for polygamma functions. Math. Slovaca 65(1), 103-120 (2015). Available online at https://doi.org/10.1515/ms-2015-0010

40. Guo, B.-N., Zhao, J.-L., Qi, F.: A completely monotonic function involving the tri- and tetra-gamma functions. Math. Slovaca 63(3), 469-478 (2013). Available online at https://doi.org/10.2478/s12175-013-0109-2

41. Ismail, M.E.H., Lorch, L., Muldoon, M.E.: Completely monotonic functions associated with the gamma function and its q-analogues. J. Math. Anal. Appl. 116, 1-9 (1986). Available online at https://doi.org/10.1016/0022-247X(86)90042-9

42. Ismail, M.E.H., Muldoon, M.E.: Inequalities and monotonicity properties for gamma and $q$-gamma functions. In: Zahar, R.V.M. (ed.) Approximation and Computation: A Festschrift in Honour of Walter Gautschi, ISNM, vol. 119, pp. 309-323. Birkhäuser, Basel (1994). A corrected version is available online at http://arxiv.org/abs/1301.1749

43. Ismail, M.E.H., Muldoon, M.E.: Inequalities and monotonicity properties for gamma and $q$-gamma functions. arXiv preprint (2013). Available online at http://arxiv.org/abs/1301.1749

44. Katriel, J.: The $q$-Lah numbers and the $n$-th $q$-derivative of $\exp _{q}(1 / x)$. Notes Number Theory Discrete Math. 23(2), 45-47 (2017)

45. Kazarinoff, D.K.: On Wallis' formula. Edinb. Math. Notes 1956(40), 19-21 (1956)

46. Koornwinder, T.H.: Jacobi functions as limit cases of $q$-ultraspherical polynomials. J. Math. Anal. Appl. 148(1), 44-54 (1990). Available online at https://doi.org/10.1016/0022-247X(90)90026-C

47. Koumandos, S.: Monotonicity of some functions involving the gamma and psi functions. Math. Comput. 77(264), 2261-2275 (2008). Available online at https://doi.org/10.1090/S0025-5718-08-02140-6

48. Koumandos, S., Lamprecht, M.: Some completely monotonic functions of positive order. Math. Comput. 79(271), 1697-1707 (2010). Available online at https://doi.org/10.1090/S0025-5718-09-02313-8

49. Koumandos, S., Lamprecht, M.: Complete monotonicity and related properties of some special functions. Math. Comput. 82(282), 1097-1120 (2013). Available online at https://doi.org/10.1090/S0025-5718-2012-02629-9

50. Koumandos, S., Pedersen, H.L.: Completely monotonic functions of positive order and asymptotic expansions of the logarithm of Barnes double gamma function and Euler's gamma function. J. Math. Anal. Appl. 355(1), 33-40 (2009). Available online at https://doi.org/10.1016/j.jmaa.2009.01.042

51. Koumandos, S., Pedersen, H.L.: Absolutely monotonic functions related to Euler's gamma function and Barnes' double and triple gamma function. Monatshefte Math. 163(1),51-69 (2011). Available online at https://doi.org/10.1007/s00605-010-0197-9 
52. Leblanc, A., Johnson, B.C.: On a uniformly integrable family of polynomials defined on the unit interval. J. Inequal. Pure Appl. Math. 8(3), Article ID 67 (2007). https://www.emis.de/journals/JPAM/article878.html

53. Li, W.-H., Qi, F., Guo, B.-N.: On proofs for monotonicity of a function involving the psi and exponential functions. Analysis (Munich) 33(1), 45-50 (2013). Available online at https://doi.org/10.1524/anly.2013.1175

54. Liu, F.-F., Shi, X.-T., Qi, F.: A logarithmically completely monotonic function involving the gamma function and originating from the Catalan numbers and function. Glob. J. Math. Anal. 3(4), 140-144 (2015). Available online at https://doi.org/10.14419/gjma.v3i4.5187

55. Mitrinović, D.S., Pečarić, J.E., Fink, A.M.: Classical and New Inequalities in Analysis. Kluwer Academic, Dordrecht (1993). Available online athttps://doi.org/10.1007/978-94-017-1043-5

56. Muldoon, M.E.: Some monotonicity properties and characterizations of the gamma function. Aequ. Math. 18, 54-63 (1978). Available online at https://doi.org/10.1007/BF01844067

57. Ouimet, F.: Complete monotonicity of multinomial probabilities and its application to Bernstein estimators on the simplex. arXiv preprint (2018). Available online at https://arxiv.org/abs/1804.02108

58. Ouimet, F: Complete monotonicity of multinomial probabilities and its application to Bernstein estimators on the simplex. J. Math. Anal. Appl. 466(2), 1609-1617 (2018). Available online at https://doi.org/10.1016/j.jmaa.2018.06.049

59. Pečarić, J.E., Proschan, F., Tong, Y.L.: Convex Functions, Partial Orderings, and Statistical Applications. Mathematics in Science and Engineering, vol. 187. Academic Press, San Diego (1992)

60. Qi, F:: Three-log-convexity for a class of elementary functions involving exponential function. J. Math. Anal. Approx. Theory 1(2), 100-103 (2006)

61. Qi, F:: A class of logarithmically completely monotonic functions and the best bounds in the first Kershaw's double inequality. J. Comput. Appl. Math. 206(2), 1007-1014 (2007). Available online at https://doi.org/10.1016/j.cam.2006.09.005

62. Qi, F.: A completely monotonic function involving the divided difference of the psi function and an equivalent inequality involving sums. ANZIAM J. 48(4), 523-532 (2007). Available online at https://doi.org/10.1017/S1446181100003199

63. Qi, F.: Three classes of logarithmically completely monotonic functions involving gamma and psi functions. Integral Transforms Spec. Funct. 18, 503-509 (2007). Available online at https://doi.org/10.1080/10652460701358976

64. Qi, F.: Bounds for the ratio of two gamma functions. J. Inequal. Appl. 2010, Article ID 493058 (2010). https://doi.org/10.1155/2010/493058

65. Qi, F.: Completely monotonic degree of a function involving the tri- and tetra-gamma functions. arXiv preprint (2013). Available online at http://arxiv.org/abs/1301.0154

66. Qi, F.: Limit formulas for ratios between derivatives of the gamma and digamma functions at their singularities. Filomat 27(4), 601-604 (2013). Available online at https://doi.org/10.2298/FIL1304601Q

67. Qi, F.: A completely monotonic function related to the q-trigamma function. Sci. Bull. "Politeh." Univ. Buchar., Ser. A Appl. Math. Phys. 76(1), 107-114 (2014)

68. Qi, F: Absolute monotonicity of a function involving the exponential function. Glob. J. Math. Anal. 2(3), 184-203 (2014). Available online at https://doi.org/10.14419/gjma.v2i3.3062

69. Qi, F: Bounds for the ratio of two gamma functions: from Gautschi's and Kershaw's inequalities to complete monotonicity. Turk. J. Anal. Number Theory 2(5), 152-164 (2014). Available online at https://doi.org/10.12691/tjant-2-5-1

70. Qi, F.: Explicit formulas for computing Bernoulli numbers of the second kind and Stirling numbers of the first kind. Filomat 28(2), 319-327 (2014). Available online at https://doi.org/10.2298/FIL14023190

71. Qi, F: Integral representations and complete monotonicity related to the remainder of Burnside's formula for the gamma function. J. Comput. Appl. Math. 268, 155-167 (2014). Available online at https://doi.org/10.1016/j.cam.2014.03.004

72. Qi, F:: Complete monotonicity of a function involving the tri- and tetra-gamma functions. Proc. Jangjeon Math. Soc. 18(2), 253-264 (2015). Available online at https://doi.org/10.17777/pjms.2015.18.2.253

73. Qi, F.: Complete monotonicity of functions involving the $q$-trigamma and $q$-tetragamma functions. Rev. R. Acad. Cienc. Exactas Fís. Nat. Ser. A Mat. 109(2), 419-429 (2015). Available online at https://doi.org/10.1007/s13398-014-0193-3

74. Qi, F.: Derivatives of tangent function and tangent numbers. Appl. Math. Comput. 268, 844-858 (2015). Available online at https://doi.org/10.1016/j.amc.2015.06.123

75. Qi, F.: Properties of modified Bessel functions and completely monotonic degrees of differences between exponential and trigamma functions. Math. Inequal. Appl. 18(2), 493-518 (2015). Available online at https://doi.org/10.7153/mia-18-37

76. Qi, F: A completely monotonic function involving the gamma and trigamma functions. Kuwait J. Sci. Eng. 43(3), 32-40 (2016)

77. Qi, F: An explicit formula for the Bell numbers in terms of the Lah and Stirling numbers. Mediterr. J. Math. 13(5), 2795-2800 (2016). Available online at https://doi.org/10.1007/s00009-015-0655-7

78. Qi, F.: Diagonal recurrence relations for the Stirling numbers of the first kind. Contrib. Discrete Math. 11, 22-30 (2016). Available online at http://hdl.handle.net/10515/sy5wh2dx6 and https://doi.org/10515/sy5wh2dx6

79. Qi, F:: A logarithmically completely monotonic function involving the q-gamma function. HAL archives (2018). Available online at https://hal.archives-ouvertes.fr/hal-01803352

80. Qi, F:: Notes on a double inequality for ratios of any two neighbouring non-zero Bernoulli numbers. Turk. J. Anal. Number Theory 6(5), 129-131 (2018). Available online at https://doi.org/10.12691/tjant-6-5-1

81. Qi, F: A double inequality for the ratio of two non-zero neighbouring Bernoulli numbers. J. Comput. Appl. Math. 351, 1-5 (2019). Available online at https://doi.org/10.1016/j.cam.2018.10.049

82. Qi, F., Berg, C.: Complete monotonicity of a difference between the exponential and trigamma functions and properties related to a modified Bessel function. Mediterr. J. Math. 10(4), 1685-1696 (2013). Available online at https://doi.org/10.1007/s00009-013-0272-2

83. Qi, F., Cerone, P., Dragomir, S.S.: Complete monotonicity of a function involving the divided difference of psi functions. Bull. Aust. Math. Soc. 88(2), 309-319 (2013). Available online at https://doi.org/10.1017/S0004972712001025 
84. Qi, F., Chapman, R.J.: Two closed forms for the Bernoulli polynomials. J. Number Theory 159, 89-100 (2016). Available online at https://doi.org/10.1016/j.jnt.2015.07.021

85. Qi, F., Chen, C.-P.: A complete monotonicity property of the gamma function. J. Math. Anal. Appl. 296(2), 603-607 (2004). Available online at https://doi.org/10.1016/j.jmaa.2004.04.026

86. Qi, F., Cui, R.-Q., Chen, C.-P., Guo, B.-N.: Some completely monotonic functions involving polygamma functions and an application. J. Math. Anal. Appl. 310(1), 303-308 (2005). Available online at https://doi.org/10.1016/j.jmaa.2005.02.016

87. Qi, F., Guo, B.-N.: Wendel's and Gautschi's inequalities: refinements, extensions, and a class of logarithmically completely monotonic functions. Appl. Math. Comput. 205(1), 281-290 (2008). Available online at https://doi.org/10.1016/j.amc.2008.07.005

88. Qi, F., Guo, B.-N.: Completely monotonic functions involving divided differences of the di- and tri-gamma functions and some applications. Commun. Pure Appl. Anal. 8(6), 1975-1989 (2009). Available online at https://doi.org/10.3934/cpaa.2009.8.1975

89. Qi, F., Guo, B.-N.: Necessary and sufficient conditions for functions involving the tri- and tetra-gamma functions to be completely monotonic. Adv. Appl. Math. 44(1), 71-83 (2010) Availale online at. https://doi.org/10.1016/j.aam.2009.03.003

90. Qi, F., Guo, B.-N.: Some properties of extended remainder of Binet's first formula for logarithm of gamma function. Math. Slovaca 60(4), 461-470 (2010). Available online at https://doi.org/10.2478/s12175-010-0025-7

91. Qi, F., Guo, B.-N.: Complete monotonicity of divided differences of the di- and tri-gamma functions with applications. Georgian Math. J. 23(2), 279-291 (2016). Available online at https://doi.org/10.1515/gmj-2016-0004

92. Qi, F., Guo, B.-N.: Integral representations and complete monotonicity of remainders of the Binet and Stirling formulas for the gamma function. Rev. R. Acad. Cienc. Exactas Fís. Nat., Ser. A Mat. 111(2), 425-434 (2017). Available online at https://doi.org/10.1007/s13398-016-0302-6

93. Qi, F., Guo, B.-N.: Integral representations of the Catalan numbers and their applications. Mathematics 5(3), Article ID 40 (2017). https://doi.org/10.3390/math5030040

94. Qi, F., Guo, B.-N.: Lévy-Khintchine representation of Toader-Qi mean. Math. Inequal. Appl. 21(2), 421-431 (2018). Available online at https://doi.org/10.7153/mia-2018-21-29

95. Qi, F., Guo, B.-N.: On the sum of the Lah numbers and zeros of the Kummer confluent hypergeometric function. Acta Univ. Sapientiae Math. 10(1), 125-133 (2018). Available online at https://doi.org/10.2478/ausm-2018-0011

96. Qi, F., Guo, B.-N.: Some properties and generalizations of the Catalan, Fuss, and Fuss-Catalan numbers. In: Ruzhansky, M., Dutta, H., Agarwal, R.P. (eds.) Mathematical Analysis and Applications: Selected Topics, 1st edn., pp. 101-133. Wiley, New York (2018). Chap. 5, Available online at https://doi.org/10.1002/9781119414421.ch5

97. Qi, F., Guo, B.-N.: The reciprocal of the weighted geometric mean of many positive numbers is a Stieltjes function. Quaest. Math. 41(5), 653-664 (2018). Available online at https://doi.org/10.2989/16073606.2017.1396508

98. Qi, F., Guo, B.-N., Chen, C.-P.: Some completely monotonic functions involving the gamma and polygamma functions. J. Aust. Math. Soc. 80(1), 81-88 (2006). Available online at https://doi.org/10.1017/S1446788700011393

99. Qi, F., Guo, B.-N., Chen, C.-P.: The best bounds in Gautschi-Kershaw inequalities. Math. Inequal. Appl. 9(3), 427-436 (2006). Available online at https://doi.org/10.7153/mia-09-41

100. Qi, F., Guo, S., Guo, B.-N.: Complete monotonicity of some functions involving polygamma functions. J. Comput. Appl. Math. 233(9), 2149-2160 (2010). Available online at https://doi.org/10.1016/j.cam.2009.09.044

101. Qi, F., Li, W.-H.: A logarithmically completely monotonic function involving the ratio of gamma functions. J. Appl. Anal. Comput. 5(4), 626-634 (2015). Available online at https://doi.org/10.11948/2015049

102. Qi, F., Li, W.H.:I Integral representations and properties of some functions involving the logarithmic function. Filomat 30(7), 1659-1674 (2016). Available online at https://doi.org/10.2298/FIL1607659Q

103. Qi, F., Lim, D.: Integral representations of bivariate complex geometric mean and their applications. J. Comput. Appl. Math. 330, 41-58 (2018). Available online at https://doi.org/10.1016/..cam.2017.08.005

104. Qi, F., Lim, D., Guo, B.-N.: Explicit formulas and identities for the Bell polynomials and a sequence of polynomials applied to differential equations. Rev. R. Acad. Cienc. Exactas Fís. Nat., Ser. A Mat. 113(1), 1-9 (2019). Available online at https://doi.org/10.1007/s13398-017-0427-2

105. Qi, F., Liu, F.-F., Shi, X.-T.: Comments on two completely monotonic functions involving the $q$-trigamma function. J. Inequal. Spec. Funct. 7(4), 211-217 (2016)

106. Qi, F., Luo, Q.-M.: Bounds for the ratio of two gamma functions-from Wendel's and related inequalities to logarithmically completely monotonic functions. Banach J. Math. Anal. 6(2), 132-158 (2012). Available online at https://doi.org/10.15352/bjma/1342210165

107. Qi, F., Luo, Q.-M.: Bounds for the ratio of two gamma functions: from Wendel's asymptotic relation to Elezović-Giordano-Pečarićs theorem. J. Inequal. Appl. 2013, 542 (2013). 20 pages, Available online at https://doi.org/10.1186/1029-242X-2013-542

108. Qi, F., Luo, Q.-M., Guo, B.-N.: Complete monotonicity of a function involving the divided difference of digamma functions. Sci. China Math. 56(11), 2315-2325 (2013). Available online at https://doi.org/10.1007/s11425-012-4562-0

109. Qi, F., Luo, Q.-M., Guo, B.-N.: The function $\left(b^{x}-a^{x}\right) / x$ : ratio's properties. In: Milovanović, G.V., Rassias, M.Th. (eds.) Analytic Number Theory, Approximation Theory, and Special Functions, pp. 485-494. Springer, Berlin (2014). Available online at https://doi.org/10.1007/978-1-4939-0258-3_16

110. Qi, F., Mahmoud, M., Shi, X.-T., Liu, F.-F.: Some Properties of the Catalan-Qi Function Related to the Catalan Numbers, vol. 5. Springer, Berlin (2016). 20 pages, Available online at https://doi.org/10.1186/s40064-016-2793-1

111. Qi, F., Mortici, C.: Some inequalities for the trigamma function in terms of the digamma function. Appl. Math. Comput. 271, 502-511 (2015). Available online at https://doi.org/10.1016/j.amc.2015.09.039

112. Qi, F., Mortici, C., Guo, B.-N.: Some properties of a sequence arising from geometric probability for pairs of hyperplanes intersecting with a convex body. Comput. Appl. Math. 37(2), 2190-2200 (2018). Available online at https://doi.org/10.1007/s40314-017-0448-7

113. Qi, F., Niu, D.-W., Lim, D., Guo, B.-N.: Some logarithmically completely monotonic functions and inequalities for multinomial coefficients and multivariate beta functions. HAL archives (2018). Available online at https://hal.archives-ouvertes.fr/hal-01769288 
114. Qi, F., Rahman, G., Hussain, S.M., Du, W.-S., Nisar, K.S.: Some inequalities of Čebyšev type for conformable k-fractional integral operators. Symmetry 10(11), Article ID 614 (2018). https://doi.org/10.3390/sym10110614

115. Qi, F., Shi, X.-T., Liu, F.-F.: An integral representation, complete monotonicity, and inequalities of the Catalan numbers. Filomat 32(2), 575-587 (2018). Available online at https://doi.org/10.2298/FIL1802575Q

116. Qi, F., Wang, S.H.: Complete monotonicity, completely monotonic degree, integral representations, and an inequality related to the exponential, trigamma, and modified Bessel functions. Glob. J. Math. Anal. 2(3), 91-97 (2014). Available online at https://doi.org/10.14419/gjma.v2i3.2919

117. Qi, F., Yao, S.-W., Guo, B.-N.: Arithmetic means for a class of functions and the modified Bessel functions of the first kind. Mathematics 7(1), Article ID 60 (2019). Available online at https://doi.org/10.3390/math7010060

118. Qi, F., Zhang, X.-J.: Complete monotonicity of a difference between the exponential and trigamma functions. J. Korea Soc. Math. Educ. Ser. B Pure Appl. Math. 21(2), 141-145 (2014). Available online at https://doi.org/10.7468/jksmeb.2014.21.2.141

119. Qi, F., Zhang, X.J., Li, W.-H.: Lévy-Khintchine representations of the weighted geometric mean and the logarithmic mean. Mediterr. J. Math. 11(2), 315-327 (2014). Available online at https://doi.org/10.1007/s00009-013-0311-z

120. Qi, F., Zhang, X.-J., Li, W.-H.: The harmonic and geometric means are Bernstein functions. Bol. Soc. Mat. Mex. (3) 23(2), 713-736 (2017). Available online at https://doi.org/10.1007/s40590-016-0085-y

121. Qi, F., Zheng, M.-M.: Explicit expressions for a family of the Bell polynomials and applications. Appl. Math. Comput. 258, 597-607 (2015). Available online at https://doi.org/10.1016/j.amc.2015.02.027

122. Schilling, R.L., Song, R., Vondraček, Z.: Bernstein Functions-Theory and Applications, 2nd edn. de Gruyter Studies in Mathematics, vol. 37. de Gruyter, Berlin (2012). Available online athttps://doi.org/10.1515/9783110269338

123. Sun, B.-C., Liu, Z.-M., Li, Q., Zheng, S.-Z.: The monotonicity and convexity of a function involving psi function with applications. J. Inequal. Appl. 2016, Article ID 151 (2016). 17 pp., available online at https://doi.org/10.1186/s13660-016-1084-2

124. Temme, N.M.: Special Functions: An Introduction to Classical Functions of Mathematical Physics. Wiley-Interscience, New York (1996). Available online at https://doi.org/10.1002/9781118032572

125. Trimble, S.Y., Wells, J., Wright, F.T.: Superadditive functions and a statistical application. SIAM J. Math. Anal. 20(5), 1255-1259 (1989). Available online at https://doi.org/10.1137/0520082

126. Wang, S.-H., Qi, F.: Hermite-Hadamard type inequalities for s-convex functions via Riemann-Liouville fractional integrals. J. Comput. Anal. Appl. 22(6), 1124-1134 (2017)

127. Widder, D.V.: The Laplace Transform. Princeton University Press, Princeton (1946)

128. Yang, Z.-H.: Some properties of the divided difference of psi and polygamma functions. J. Math. Anal. Appl. 455(1), 761-777 (2017). Available online at https://doi.org/10.1016/j.jmaa.2017.05.081

129. Yang, Z.-H., Chu, Y.-M.: Monotonicity and absolute monotonicity for the two-parameter hyperbolic and trigonometric functions with applications. J. Inequal. Appl. 2016, Article ID 200 (2016). 10 pp., available online at https://doi.org/10.1186/s13660-016-1143-8

130. Yang, Z.-H., Chu, Y.-M., Tao, X.-J.: A double inequality for the trigamma function and its applications. Abstr. Appl. Anal. 2014, Article ID 702718 (2014). https://doi.org/10.1155/2014/702718

131. Yang, Z.-H., Tian, J.-F.: A comparison theorem for two divided differences and applications to special functions. J. Math. Anal. Appl. 464, 580-595 (2018). Available online at https://doi.org/10.1016/j.jmaa.2018.04.024

132. Yang, Z.-H., Tian, J.F.: A class of completely mixed monotonic functions involving the gamma function with applications. Proc. Am. Math. Soc. 146(11), 4707-4721 (2018). Available online at https://doi.org/10.1090/proc/14199

133. Yang, Z.-H., Zheng, S.-Z.: Complete monotonicity involving some ratios of gamma functions. J. Inequal. Appl. 2017, Article ID 255 (2017). 17 pp., Available online at https://doi.org/10.1186/s13660-017-1527-4

134. Zhang, X.-J., Qi, F., Li, W.-H.: Properties of three functions relating to the exponential function and the existence of partitions of unity. Int. J. Open Probl. Comput. Sci. Math. 5(3), 122-127 (2012). Available online at https://doi.org/10.12816/0006128

135. Zhao, J.-L.: A completely monotonic function relating to the $q$-trigamma function. J. Math. Inequal. 9(1), 53-60 (2015). Available online at https://doi.org/10.7153/jmi-09-05

136. Zhao, J.-L., Guo, B.-N., Qi, F.: Complete monotonicity of two functions involving the tri- and tetra-gamma functions. Period. Math. Hung. 65(1), 147-155 (2012). Available online at https://doi.org/10.1007/s10998-012-9562-x

\section{Submit your manuscript to a SpringerOpen ${ }^{\circ}$ journal and benefit from:}

- Convenient online submission

- Rigorous peer review

- Open access: articles freely available online

- High visibility within the field

- Retaining the copyright to your article

Submit your next manuscript at $\gg$ springeropen.com 